\title{
MiR-26a-1 Promotes DNA Damage Repair by Inhibiting Sirt1 and KDM5A in Human Liver Cancer Stem Cells
}

\section{Liyan Wang}

Tongji University

Xiaonan Li

Tongji University

Rushi Qin

Tongji University

Yanan Lu

Tongji University

shuting Song

Tongji University

\section{Yingjie Chen}

Tongji University

Sijie Xie

Tongji University

Xiaoxue Jiang

Tongji University

Dongdong Lu ( $\square$ ludongdong@tongji.edu.cn )

Tongji University https://orcid.org/0000-0002-7417-4172

\section{Research}

Keywords: MiR-26a-1, DNA damage repair, Sirt1, KDM5A, liver cancer stem cells

Posted Date: March 1st, 2021

DOI: https://doi.org/10.21203/rs.3.rs-251571/v1

License: (c) (i) This work is licensed under a Creative Commons Attribution 4.0 International License.

Read Full License 


\section{Abstract}

Background: Although miR-26a-1 was down-regulated expressin in several cancers, the role of miR-26a1 in malignancies has yet to be systematically elucidated.

Methods: RT-PCR, Western blotting and tumorigenesis test in vitro and in vivo were performed to analyze the signaling pathway.

Results: miR-26a-1 inhibits the NAD(+)-dependent deacetylase Sirt1 expression by targeting the 3' noncoding region of Sirt1 which enhances the acetylation modification of $\mathrm{H} 4$ on the 16th lysine of histone and the expression of protein arginine methyltransferase PRMT6. Therefore, miR-26a-1 promotes arginine methylation modification of POLB (R137) and histone. On the other hand, miR-26a-1 inhibits the expression of KDM5A by targeting its 3 ' non-coding region, which enhances the methylation modification of histone $\mathrm{H} 3$ ysine 4. Moreover, miR-26a-1 enhances the expression of histone methyltransferase SETD2 dependent on H3K4me3 and further increases the trimethylation modification of the histone $\mathrm{H} 3$ lysine 36 . Significantly, miR-26a-1 promotes the formation of DNA damage repair complex (Rad51-PARP1-ATRATM-hMSH6-XRCC-POLB-SKP2) via H3K36me3. In particular, it was found that miR-26a-1 inhibited the function of long non-coding RNA HULC and promoted the formation of DNA damage repair complex. Furthermore, miR-26a-1 promotes the DNA damage repair ability by promoting the DNA damage repair complex to bind to the DNA damage site, thereby inhibiting the DNA damage of liver cancer stem cells. In particular, miR-26a-1 enhanced the binding of H3F3A to Skp2, CUL1, and F-box at the DNA damage site and enhanced the protein ubiquitination modification of H3F3A, which promoted Histone $\mathrm{H} 3$ replaces H3F3A by degrading H3F3A, realizing the renewal of histones after DNA damage repair. It was further found that miR-26a-1 inhibited the formation and instability of DNA microsatellites by promoting DNA damage repair, thereby affecting the expression of several cyclins and protein kinases in liver cancer stem cells, such as, inhibiting CDK2 and CyclinE , CDK4, CyclinD1, CDK6, CDK8, CyclinM2, CDK15, pRB, PCNA, MAP3K2, PGK1 and promoting RB, P18, P21/WAF1/Cip1, and thus inhibited the growth of liver cancer stem cells. Strikingly, the rescued-test further confirmed that excessive Sirt1 and KDM5A abrogated the oncogenic function of miR-26a-1. Conclusions: miR26a-1 may acts as the potential biomarker and therapeutic target for liver cancer.

\section{Background}

Although miR-26a-1 was down-regulated expressin in several cancers, the role of miR-26a-1in malignancies has yet to be systematically elucidated. miR-26 suppresses adipocyte progenitor differentiation and fat production by targeting Fbxl19 and miR-26 blocks adipogenesis, at least in part, by repressing expression of $F b x / 19$ (1). RNA G-quadruplexes (RG4s) appears to be important in posttranscriptional gene regulation, highlighting an important role of RG4 in physiology and pathology(2). miR26a protected VSMCs against H2O2induced injury through activation of the PTEN/AKT/mTOR pathway, and miR26a may be considered as a potential prognostic biomarker and therapeutic target in the treatment of AAA injury through activation of the PTEN/AKT/mTOR pathway(3). knockout of miR26a 
increased surfactantassociated mRNA and protein expression levels(4). In particular, Expression of miR26a exhibits a negative correlation with HMGA1 and regulates cancer progression by targeting HMGA1 in lung adenocarcinoma cells (5).

DNA damage repair plays a important role in hepatocarcinigenesis. The decision between cell survival and death following DNA damage rests on factors that are involved in DNA damage recognition, as well as on factors involved in the activation of apoptosis, autophagy (6). Deviations in this fine-tuning are known to destabilize cellular metabolic homeostasis, as exemplified in diverse cancers where disruption or deregulation of DNA repair pathways results in genome instability(7). DNA repair factors ultimately contribute to DNA repair pathway choice between homologous recombination and non-homologous end joining(8).Cancer chemotherapy and radiotherapy are designed to kill cancer cells mostly by inducing DNA damage. (9).DNA damage repair systems have evolved to act as a genome-wide surveillance mechanism to maintain chromosome integrity and impairment of these systems gives rise to mutations and directly contributes to tumorigenesis(10).

In the study, miR-26a-1 inhibits the expression of NAD+-dependent deacetylase Sirt1 and KDM5A, thereafter, miR-26a-1 promotes DNA damage repair, thereby affecting the expression of some cyclins and protein kinases in liver cancer stem cells. Moreover, excessive Sirt1 and KDM5A abolished the oncogenic functions of miR-26a-1 in liver cancer stem cells. In conclusions, miR26a-1 may acts as the potential biomarker and therapeutic target for liver cancer. We also shed light on the fact that the attenuation of deregulated functioning of miRNA could be a viable approach for cancer treatment.

\section{Materials And Methods}

CD133+/CD44 + Huh7 cells sorting CD133/CD44 MicroBead Kits were purchased from Miltenyi technic(Boston, USA) and MACS ${ }^{\circledR}$ Technology operation according to and the operation according to the manufacturer.

Cell Lines Cells were maintained in Dulbecco's modified Eagle medium(Gibco BRL Life Technologies) supplemented with $10 \%$ heat-inactivated $\left(56^{\circ} \mathrm{C}, 30\right.$ minutes) fetal bovine serum (sigma) in a humidified atmosphere of $5 \% \mathrm{CO}_{2}$ incubator at $37^{\circ} \mathrm{C}$.

RT-PCR cDNA was prepared by using oligonucleotide (dT), random primers, and a SuperScript First-Strand Synthesis System (Invitrogen). PCR analysis was performed according to the manufacturer. $\beta$-actin was used as an internal control.

Western Blotting Proteins were separated on a 10\% sodium dodecyl sulfate-polyacrylamide gel electrophoresis (SDS-PAGE) and transferred onto a nitrocellulose membranes (Invitrogen). And then blocked in $10 \%$ dry milk-TBST (20mM Tris-HCl [PH 7.6], $127 \mathrm{mM} \mathrm{NaCl}, 0.1 \%$ Tween 20 ) for $1 \mathrm{~h}$ at $37^{\circ} \mathrm{C}$. Following three washes in Tris- $\mathrm{HCl} \mathrm{pH} 7.5$ with $0.1 \%$ Tween 20 , the blots were incubated with antibody(appropriate dilution) overnight at $4^{\circ} \mathrm{C}$. Signals were visualized by enhanced chemiluminescence plus kit(GE Healthcare). 
RNA Immunoprecipitation(RIP) Ribonucleoprotein particle-enriched lysates were incubated with protein G/A-plus agarose beads (Santa Cruz) together with antibody or normal rabbit IgG for 4 hours at $4^{\circ} \mathrm{C}$. Beads were subsequently washed. RNAs were isolated and then RT-PCR.

Super-RNA-EMSA Cells were washed and scraped in ice-cold PBS to prepare nuclei for electrophoretic gel mobility shift assay with the use of the gel shift assay system (Promega) modified according to the manufacturer's instructions.

CHIP assay Cells were cross-linked with 1\% (v/v) formaldehyde (Sigma) for 10 min at room temperature and stopped with $125 \mathrm{~mm}$ glycine for $5 \mathrm{~min}$. Crossed-linked cells were washed with phosphate-buffered saline, resuspended in lysis buffer, and sonicated for 8-10 $\mathrm{min}$ in a SONICS VibraCell to generate DNA fragments. Chromatin extracts were diluted 5-fold with dilution buffer, pre-cleared with Protein-A/GSepharose beads, and immunoprecipitated with specific antibody on Protein-A/G-Sepharose beads. After washing, elution and de-cross-linking, the ChIP DNA was detected by PCR.

DNA damage repair assay DNA damage marker $\mathrm{rH} 2 \mathrm{AX}$ (S139) detection, in situ DNA damage analysis and Quantitative analysis of DNA Damgae via 8-OHdG were performed according to the manufacturer's instructions, respectively.

Cell colony-formation efficiency assay. cells were plated in six wells and incubated in a humidified atmosphere of $5 \% \mathrm{CO}_{2}$ incubator at $37^{\circ} \mathrm{C}$ for 14 days. For visualization, colonies were stained with $0.5 \%$ Crystal Violet (sigma) in 50\% methanol and 10\% glacial acetic acid. Colonies were counted using a dissecting microscope by MacBiophotonics Image $\mathrm{J}$.

Tumorigenesis testin vivo Four-weeks male athymic Balb/c mice were maintained in the Tongji university animal facilities approved by the China Association for accreditation of laboratory animal care. athymic Balb/c mice per group were injected at the armpit area subcutaneously with cells. The mice were observed over 4 weeks for tumor formation. The mice were then sacrificed and the tumors recovered. The wet weight of each tumor was determined for each mouse. A portion of each tumor was fixed in $4 \%$ paraformaldehyde and embedded in paraffin for histological examination.

\section{Results}

\section{miR-26a-1 inhibits the growth of liver cancer stem cells}

To investigate the effect of miR-26a-1 on human liver cancer stem cells, CD133/CD44/CD24/EpCAM microbeads (MicroBeads) were used to isolate liver cancer stem cells from the Huh7 cell line (Figure S1A). CD133, CD44, CD24 and EpCAM were expressed in hLCSCs cells (Figure S1B\&C). Moreover, the sphere and xenograft tumor $(0.657 \pm 0.193$ gram vs $0, n=10, p=0.00000067<0.01)$ are produced in hLCSCs group, but not in non-hLCSCs group(Figure S1D\&E). In human hLCSCs transfected with rLV, rLVmiR-26a-1, rLV-Cas9, rLV-Cas9-miR-26a-1 (Fig. 1A), miR-26a-1 was significantly increased in the rLV-miR26a-1 group compared with the rLV group and knocked out in rLV-Cas9- miR-26a-1 group Compared with 
the rLV-Cas9 group (Fig. 1B-F). The cell grow ability was significantly decreased in the rLV-miR-26a-1 group compared with the rLV group ( 24 hours: $P=0.0054<0.01$; 48 hours: $P=0.00259<0.01$ ) and increased in rLV-Cas9-miR-26a-1 group Compared with the rLV-Cas9 group (24th hour: $\mathrm{P}=0.001533<$ 0.01 ; 48 hours: $P=0.003099<0.01$ ) (Fig. $1 G$ ). The positive rate of BrdU was significantly decreased in the rLV-miR-26a-1 group compared with the rLV group $(36.93 \pm 4.33 \%$ vs $19.27 \pm 2.19 \%, P=0.0192<0.01)$ and increased in rLV-Cas9-miR-26a-1 group Compared with the rLV-Cas9 group ( $40.75 \pm 4.26 \%$ vs $70.59 \pm$ $7.96 \%, P=0.0028909<0.01$ ) (Fig. $1 \mathrm{H})$. The colony formation ability was significantly decreased in the rLV-miR-26a-1 group compared with the rLV group $(42.34 \pm 3.01 \%$ vs $11.15 \pm 2 \%, P=0.0003451<0.01)$ and increased in rLV-Cas9-miR-26a-1 group compared with the rLV-Cas9 group (43.67 $\pm 3.85 \%$ vs $81.12 \pm$ $7.28 \%, P=0.00576<0.01$ ) (Fig. 1I). The sphere formation rate was significantly decreased in the rLV-miR$26 \mathrm{a}-1$ group compared with the rLV group $(59.64 \pm 6.27 \%$ vs $23.89 \pm 2.69 \%, P=0.00793<0.01)$ and increased in rLV-Cas9-miR-26a-1 group compared with the rLV-Cas9 group ( $64.82 \pm 8.65 \%$ vs $64.82 \pm$ $8.65 \%, P=0.009971<0.01$ ) (Fig. $1 \mathrm{~J}$ ). The average weight of xenograft tumors was significantly decreased in the rLV-miR-26a-1 group compared with the rLV group $(0.51 \pm 0.065 \mathrm{~g}$ vs $0.16 \pm 0.0374 \mathrm{~g}, \mathrm{P}=$ $0.00000534<0.01)$ and increased in rLV-Cas9-miR-26a-1 group compared with the rLV-Cas9 group (0.46 \pm 0.0516 grams vs $0.9714 \pm 0.2333$ grams, $P=0.000579<0.01$ ) (Fig. $1 \mathrm{~K} \& \mathrm{~L}$ ). The average appearance time of xenograft tumors was significantly increased in the rLV-miR-26a-1 group compared with the rLV group $(8.86 \pm 0.69$ days vs $14.86 \pm 1.86$ days, $P=0.0000232<0.01)$ and decreased in rLV-Cas9-miR-26a-1 group compared with the rLV-Cas9 group $(9.29 \pm 1.112$ days vs $6.143 \pm 0.8997$ days, $P=<0.01)$ (Fig. $1 \mathrm{M})$. The poorly differentiated cancer cells in transplanted tumor tissues was reduced in the rLV-miR-26a-1 group compared with the rLV group and increased in rLV-Cas9-miR-26a-1 group compared with the rLVCas9 group (1N). PCNA expression was reduced in the rLV-miR-26a-1 group compared with the rLV group $(34.21 \pm 9.403 \%$ vs $11.22 \pm 2.86 \%, P=0.000834<0.01$ ) and increased in rLV-Cas9-miR-26a-1 group compared with the rLV-Cas9 group $(30.45 \pm 3.48 \%$ vs $67.46 \pm 5.09 \%, p=0.0000042048<0.01)$

(Fig. 10a\&b). Collectively, these results suggest that miR-26a-1 inhibits the growth of human liver cancer stem cells[Also see and Supplemental Resuts:miR-26a-1 inhibits the growth of human liver cancer stem cells (FigureS2A-0)].

\section{miR-26a-1 targets NAD+-dependent deacetylase Sirt1 and enhances the acetylation modification of histone H4 lysine 16}

To clarify the effect of miR-26a-1 on human liver cancer stem cell lines (hLCSCs), we will analyze whether miR-26a-1 targets NAD+-dependent deacetylase Sirt1 and enhances the acetylation modification of histone $\mathrm{H} 4$ lysine 16. miR-26a-1 was significantly increased in rLV-miR-26a-1 group compared with rLV group and drecreaed rLV-Cas9-miR-26a-1 group compared with rLV-Cas9 group (Fig. 2A-D).

Bioinformatics analysis showed that the mature sequence of miR-26a-1 was linked to Sirt1 mRNA 3'noncoding region through 13 base complementary seed sequences (UTR) (1153-1159) (Fig. 2E). The pEZX-MT-Sirt1 3'-UTR-Luc luciferase reporter gene activity was significantly reduced in the rLV-miR-26a-1 group compared with the rLV group $(20029.1 \pm 1136.69$ vs $3781.82 \pm 474.12, P=0.0014437<0.01)$ and was significantly increased in the rLV-Cas9-miR-26a-1 groupcompared with the rLV-Cas9 group (21843.3 \pm 1556.64 vs $38605.05 \pm 4454.28, P=0.006014<0.01$ ) (Fig. 2F). The pEZX-MT-Sirt1 3'-UTR (mutant)-Luc 
luciferase reporter gene activity was not significantly Changed in rLV-miR-26a-1 group compared with the rLV group (18573.59 \pm 2743.32 vs $20258.26 \pm 1196.502, P=0.1948>0.05)$ and in rLV-Cas9-miR-26a- 1 group compared with rLV-Cas9 group (20769.59 \pm 2369.82 vs $19305.63 \pm 1167.56, P=0.273944>0.05)$ (Fig. 2G). Although the transcription level of Sirt1 was not significantly altered (Fig. 2H), the level of translation of Sitr1 was significantly reduced in the rLV-miR-26a-1 group compared with the rLV group and increased in the rLV-Cas9-miR-26a-1 group compared with the rLV-Cas9 group (Fig. 2I). The level of H4K16Ac was significantly increased in the rLV-miR-26a-1 group compared with the rLV group increased in the rLV-Cas9-miR-26a-1 groupcompared with the rLV-Cas9 group (Fig. 2J). However, the level of H4K16Ac was not significantly altered in hLCSCs in the rLV-miR-26a-1 + rLV-Sirt1 group compared with the rLV group (Fig. 2K). Collectively, these results suggest that miR-26a-1 enhances the acetylation modification of histone $\mathrm{H} 4$ lysine 16 by reducing Sirt1[Also see and Supplemental Resuts: miR-26a-1 targets NAD+-dependent deacetylase Sirt1 in human liver cancer stem cells and enhances the acetylation modification of histone $\mathrm{H} 4$ lysine 16 (FigureS3A-L)].

\section{miR-26a-1 enhances the expression of protein arginine methyltransferase PRMT6}

Given that miR-26a-1 increases the acetylation modification of histone $\mathrm{H} 4$ lysine 16 , we will further investigate whether miR-26a-1 enhances protein PRMT6 in liver cancer stem cells. The loading of H4K16Ac with PRMT6 promoter was significantly enhanced in the rLV-miR-26a-1 group compared with the rLV group and weakened in the rLV-Cas9-miR-26a-1 group compared with the rLV-Cas9 group (Fig. 3A). The binding ability of H4K16Ac with the PRMT6 promoter probe was significantly enhanced in the rLV-miR-26a-1 group compared with the rLV group and drcreaed in the rLV-Cas9-miR-26a-1 group compared with the rLV-Cas9 group (Fig. 3B). The ability of H4K16Ac and RNA Polll to enter the PRMT6 promoter-enhancer loop was significantly increased in the rLV-miR-26a-1 group compared with the rLV group and weakened in the rLV-Cas9-miR-26a-1 group compared with the rLV-Cas9 group (Fig. 3C). However, the ability of H4K16Ac and RNA Polll to enter the PRMT6 promoter-enhancer loop was not significantly changed in the rLV-miR-26a-1 + H4K16Ac inhibitor group compared with the rLV group (Fig. 3D). The activity of the pEZX-MT-PRMT6 promoter-Luc luciferase activity was significantly increased in the rLV-miR-26-1 group compared with the rLV group (77457.45 \pm 10051.33 vs $270650.41 \pm 41120.78$, $\mathrm{P}=0.005127<0.01)$ and reduced rLV-Cas9-miR-26a-1 group compared to rLV-Cas9 group $(74895.04 \pm$ 6114.57 vs $10770.04 \pm 1695.32, P=<0.000892510 .01$ ) (Fig. 3E). Although the pEZX-MT-PRMT6 promoter-Luc luciferase activity was significantly increased in the rLV-miR-26-1 group compared with the rLV grouprLV-miR-26-1 group was significantly increased (59240.21 \pm 9355.84 vs $167876.6 \pm 11085.01, P$ $=0.0009681<0.01)$, however, it was not significantly altered in the rLV-miR-26a- $1+$ rLV-Sirt1 group compared with rLV group ( $59240.21 \pm 9355.84$ vs $51853.19 \pm 3551.72, \mathrm{P}=0.11984>0.051)$ (Fig. 3F). The expression level of PRMT6 was significantly increased in the rLV-miR-26a-1 group compared with the rLV group and decreased in the rLV-Cas9-miR-26a-1 group compared with the rLV-Cas9 group (Fig. 3G\&H). However, the expression level of PRMT6 was not significantly alteed in the rLV-miR-26a-1 + rLV-Sirt1 group compared with the rLV group (Fig. 3I\&J). Collectively, these results suggest that miR-26a-1 enhance the expression of PRMT6 gene dependente on H4K16Ac[Also see and Supplemental Resuts: miR-26a-1 enhances the expression of protein arginine methyltransferase PRMT6(FigureS4A-J)]. 


\section{miR-26a-1 promotes the methylation modification of POLB (R137) and histone arginine through PRMT6}

Given that miR-26a-1 promotes the expression of PRMT6, we will analyze whether miR-26a-1 affects the methylation modification of POLB(R137) and histone arginine through PRMT6 in liver cancer stem cells. The expression of POLB was not significantly altered in the rLV-miR-26a-1 group compared with the rLV group and in the rLV-Cas9-miR-26a-1 group compared with the rLV-Cas9 group (Fig. 4A). The interaction between POLB and PRMT6 was significantly increased in the rLV-miR-26a-1 group compared with the rLV group and decreased in the rLV-Cas9-miR-26a-1 group compared with the rLV-Cas9 group (Fig. 4B). The level of methylation modification of POLB (R137) was significantly increased in the rLV-miR-26a-1 group compared with the rLV group and decreased in the rLV-Cas9-miR-26a-1 group compared with the rLVCas9 group (Fig. 4C). However, the methylation modification level of PPOLB (R137) was not significantly changed in the hLCSCs of the rLV-miR-26a-1 + pGFP-V-RS-PRMT6 group compared with the rLV group (Fig. 4D). The interaction of H2A, H3, H4 with PRMT6 was significantly increased in the rLV-miR-26a-1 group compared with the rLV group and decreased in the rLV-Cas9-miR-26a-1 group compared with the rLV-Cas9 group (Fig. 4E). The modification level of H3R2me2, H2AR3me, H4R3me was significantly increased in the rLV-miR-26a-1 group compared with the rLV group and decreased in the rLV-Cas9-miR26a-1 group compared with the rLV-Cas9 group (Fig. 4F). However, the H3R2me2, H2AR3me and H4R3me were not significantly changed in the rLV-miR-26a-1 + pGFP-V-RS-PRMT6 group compared with the rLV group (Fig. 4G). Collectively, these results suggest that miR-26a-1 enhances the methylation modification of POLB(R137) and histone arginine through PRMT6[Also see and Supplemental Resuts: miR-26a-1 promotes the methylation modification of POLB (R137) and histone arginine through PRMT6(FigureS5A$\mathrm{G})$.

\section{miR-26a-1 enhances the methylation modification of histone H3 lysine 4 via targeting KDM5A}

In order to study whether miR-26a-1 affects the expression of KDM5A and the methylation modification of histone $\mathrm{H} 3$ lysine 4, we conducted related experiments. The mature miR-26a-1 binds to KDM5A "mRNA" 3'-uncoding region (UTR) (4606-4612) through an 11-base complementary seed sequence(Fig. 5A). The pEZX-MT-KDM5A 3'-UTR-Luc luciferase reporter gene was significantly reduced in the rLV-miR-26a-1 group compared with the rLV group $(23493.59 \pm 2166.57$ vs6301.64 $\pm 829.17, \mathrm{P}=0.00108<0.01)$ and enhanced in the rLV-Cas9-miR-26a-1 group compared with the rLV-Cas9 group (30661.08 $\pm 2077.47 \mathrm{vs}$ $88263.9 \pm 9474.02, \mathrm{P}=0.003246<0.01$ ) (Fig. 5B). The pEZX-MT-KDM5A 3'-UTR(mutant)-Luc luciferase reporter gene activity was not significantly altered in rLV-miR-26a-1 group compared with the rLV group (31943.78 \pm 3240.52 vs $31529.303 \pm 1797.79, P=0.437>0.05)$ and in the rLV-Cas9-miR-26a-1 group compared with the rLV-Cas 9 group $(34014.637 \pm 2880.78$ vs $34666.77 \pm 4845.201, P=0.415>0.05)$ (Fig. 5C). The transcription level of KDM5A was not significantly altered in rLV-miR-26a-1 group compared with the rLV group and in the rLV-Cas9-miR-26a-1 group compared with the rLV-Cas9 group (Fig. 5D). The expression of KDM5A was significantly reduced in rLV-miR-26a-1 group compared with the rLV group and increased in the rLV-Cas9-miR-26a-1 group compared with the rLV-Cas9 group (Fig. 5E). The level of H3K4me3 was significantly increased in rLV-miR-26a-1 group compared with the rLV group and decreased in the rLV-Cas9-miR-26a-1 group compared with the rLV-Cas9 group (Fig. 5F). However, the 
level of H3K4me3 was not significantly altered in the rLV-miR-26a-1 + rLV-KDM5A group compared with the rLV group (Fig. 5G). Collectively, these results suggest that miR-26a-1 enhances the methylation modification of histone $\mathrm{H} 3$ lysine 4 by reducing KDM5A[Also see and Supplemental Resuts: miR-26a-1 enhances the methylation modification of histone H3 lysine 4 via targeting KDM5A (FigureS6A-G)].

\section{miR-26a-1 enhances the expression of histone methyltransferase SETD2 and the trimethylation modification on the 36th lysine of Histone H3 dependent on H3K4me3}

Given that miR-26a-1 increases the trimethylation modification of histone $\mathrm{H} 3$ lysine 4, we will further investigate whether miR-26a-1 affects SETD2 and H3K36me3 dependent on H3K4me3. The binding ability of $\mathrm{H} 3 \mathrm{~K} 4 \mathrm{me} 3$ to the histone methyltransferase SETD2 promoter was significantly enhanced in the rLV-miR-26a-1 group compared with the rLV group and reduced in the rLV-Cas9-miR-26a-1 group compared with the rLV-Cas9 group (Fig. 6A). The binding ability of H3K4me3 to the histone methyltransferase SETD2 promoter probe was significantly enhanced in the rLV-miR-26a-1 group compared with the rLV group and reduced in the rLV-Cas9-miR-26a-1 group compared with the rLV-Cas9 group (Fig. 6B). The ability of H3K4me3 and RNA Polll to enter the histone methyltransferase SETD2 promoter-enhancer loop was significantly increased in the rLV-miR-26a-1 group compared with the rLV group and reduced in the rLV-Cas9-miR-26a-1 group compared with the rLV-Cas9 group (Fig. 6C). However, it was not significantly altered in the rLV-miR-26a-1 + rLV-KDM5A group compared with the rLV group (Fig. 6D). The activity of the pEZX-MT-SETD2 promoter-Luc luciferase reporter gene was significantly increased in the rLV-miR-26a-1 group compared with the rLV group (316758.34 \pm 45109.35 vs $1781523.14 \pm 99201.06, P=0.00044<0.01)$ and reduced in the rLV-Cas9-miR-26a-1 group compared with the rLV-Cas9 group (368253.56 \pm 75472.56 vs $47702.19 \pm 9066.13, P=00709<0.01)$ (Fig. 6E). However, it was not significantly altered in the rLV-miR-26a-1 + rLV-KDM5A group compared with the rLV group ( $551892.38 \pm 112067.76$ vs $677012.65 \pm 78394.74, \mathrm{P}=0.06122>0.051)$ (Fig. 6F). The expression of SETD2 was significantly increased in the rLV-miR-26a-1 group compared with the rLV group and reduced in the rLV-Cas9-miR-26a-1 group compared with the rLV-Cas9 group (Fig. 6G\&H). However, it was not significantly altered (Fig. 6I\&J). The interaction between histone $\mathrm{H} 3$ and SETD2 was significantly increased in the rLV-miR-26a-1 group compared with the rLV groupand reduced in the rLV-Cas9-miR-26a-1 group compared with the rLV-Cas9 group (Fig. 6K). The H3K36me3 was significantly increased in the rLVmiR-26a-1 group compared with the rLV group and reduced in the rLV-Cas9-miR-26a-1 group compared with the rLV-Cas9 group (Fig. 6L). However, it was not significantly altered in the rLV-miR-26a-1 + pGFP-VRS-SETD2 group compared with the rLV group (Fig. 6M). Collectively, these results suggest that miR-26a1 enhances the expression of histone methyltransferase SETD2 and the trimethylation modification on the 36th lysine of Histone H3 dependent on H3K4me3[Also see and Supplemental Resuts: miR-26a-1 enhances the expression of histone methyltransferase SETD2 and the trimethylation modification on the 36th lysine of Histone H3 dependent on H3K4me3 (FigureS7A-O)].

miR-26a-1 promotes the formation of DNA damage repair complex dependent on the H3K36me3 
Given that miR-26a-1 promotes the trimethylation of histone $\mathrm{H} 3$ on lysine 36 , we will analyze whether miR-26a-1 to promote DNA damage repair complex formation dependent on the trimethylation of histone H3 on lysine 36. The binding ability of H3K36me3 with Rad51, PARP1, ATR, ATM, hMSH6, XRCC5, POLB, and SKP2 was significantly increased in the rLV-miR-26a-1 group compared with the rLV group and reduced in the rLV-Cas9-miR-26a-1 group compared with the rLV-Cas9 group(Fig. 7A). The binding of mismatched DNA damage probes to Rad51, PARP1, ATR, ATM, hMSH6, XRCC5, POLB, SKP2 was significantly increased in the rLV-miR-26a-1 group compared with the rLV group and reduced in the rLVCas9-miR-26a-1 group compared with the rLV-Cas9 group (Fig. 7B). However, the H3K36me3 was significantly reduced in the rLV-miR-26a-1 + rLV-KDM4A group compared with the rLV group (Fig. 7C). The binding ability of Rad51, PARP1, ATR, ATM, hMSH6, XRCC5, POLB, and SKP2 to mismatched DNA damage probes was not significantly altered in the rLV-miR-26a- $1+$ rLV-KDM4A group compared with the rLV group (Fig. 7D). Next, Transfect the mismatched plasmid was transfected(Fig. 7E) and then perform repeated chromatin immunoprecipitation (CHIP) to analyze the binding ability of the methylated POLB with the mismatched sequence. The results showed that the binding ability of the methylated POLB to the mismatched DNA sequence was significantly enhanced in the rLV-miR-26a-1 group compared with the rLV group and reduced in the rLV-Cas9-miR-26a-1 group compared with the rLV-Cas9 group (Fig. 7F). The binding ability of mismatched DNA sequences to Rad51, PARP1, ATR, ATM, hMSH6, XRCC5, POLB, SKP2 was significantly enhanced in the rLV-miR-26a-1 group compared with the rLV group and reduced in the rLV-Cas9-miR-26a-1 group compared with the rLV-Cas9 group ( Fig. 7G). Collectively, these results suggest that miR-26a-1 enhances the binding ability of Rad51, PARP1, ATR, ATM, hMSH6, XRCC5, POLB, SKP2 to mismatch DNA damage dependent on H3K36me3, and promotes the formation of DNA damage repair complexes. [Also see and Supplemental Resuts: miR-26a-1 promotes the formation of DNA damage repair complex dependent on the H3K36me3 (FigureS8A-F)].

\section{miR-26a-1 promotes the formation of DNA damage repair complexes dependent on long non-coding RNA HULC}

In order to investigate whether miR-26a-1 promotes the formation of DNA damage repair complexes, we first analyzed whether non-coding RNA HULC is related to the formation of DNA damage repair complexes. HULC was significantly increased in the rLV-HULC group compared with the rLV group and reduced in the pGFP-V-RS-HULC group compared with the pGFP-V-RS group (Fig. 8A). The binding ability of PARP1, Rad51 to HULC was significantly enhanced in the rLV-HULC group compared with the rLV group and reduced in the pGFP-V-RS-HULC group compared with the pGFP-V-RS group (Fig. 8B). Compared with the rLV group, in the rLV-HULC group, The binding ability of H3K36me3, Rad51, PARP1, ATR, ATM, hMSH6, XRCC5, POLB, SKP2 to mismatched DNA damage probes was significantly reduced in the rLV-HULC group compared with the rLV group and enhanced in the pGFP-V-RS-HULC group compared with the pGFP-V-RS group (Fig. 8C). Compared with the rLV group, The binding ability of H3K36me3, Rad51, PARP1, ATR, ATM, hMSH6, XRCC5, POLB, SKP2 to mismatched DNA sequences was significantly weakened in the rLV-HULC group compared with the rLV group and increased in the pGFP-V-RS-HULC group compared with the pGFP-V-RS group (Fig. 8D).The mutual binding ability between PARP1, Rad51 to HULC was significantly reduced in the rLV-HULC group compared with the rLV group and increased in the 
pGFP-V-RS-HULC group compared with the pGFP-V-RS group (Fig. 8E).The expression of HULC was not significantly altered in the rLV-miR-26a-1 group compared with the rLV group, however, it was significantly increased in the rLV-miR-26a-1 + rLV-HULC group (Fig. 8F). The binding capacity of H3K36me3, Rad51, PARP1, ATR, ATM, hMSH6, XRCC5, POLB, and SKP2 to mismatched DNA damage probes was significantly increased in the rLV-miR-26a-1 group compared with the rLV group, however, it was not significantly changed in the rLV-miR-26a-1 + rLV-HULC group compared with the rLV group (Fig. 8G). Compared with the rLV group, The ability of Rad51, PARP1, ATR, ATM, hMSH6, XRCC5, POLB, SKP2 to mismatched DNA sequences were significantly enhanced in the rLV-miR-26a-1 group compared with the rLV group, however, it was not significantly altered in the rLV-miR-26a-1 + rLV-HULC group compared with the rLV group (Fig. 8H). Collectively, these results suggest that that miR-26a-1 promotes the formation of DNA damage repair complexes dependent on long non-coding RNA HULC in liver cancer stem cells. [Also see and Supplemental Resuts: miR-26a-1 promotes the formation of DNA damage repair complexes dependent on long non-coding RNA HULC (FigureS9A-C)].

\section{miR-26a-1 promotes DNA damage repair in human liver cancer stem cells}

Given that miR-26a-1 promotes the formation of the DNA damage repair complex, we will analyze whether miR-26a-1 affects the DNA damage repair in liver cancer stem cells. rH2AX (S139) was significantly reduced in the rLV-miR-26a-1 group compared with the rLV group and increased in the rLVCas9-miR-26a-1 group compared with the rLV-Cas9 group (Fig. 9A). However, it was not significantly alterd in the hLCSCs of the rLV-miR-26a-1 + rLV-KDM5A group compared with the rLV group (Fig. 9B). rH2AX (S139) was significantly reduced in the rLV-miR-26a-1 group compared with the rLV group and increased in the rLV-Cas9-miR-26a-1 group compared with the rLV-Cas9 group (Fig. 9C). However, it was not significantly altered in the rLV-miR-26a-1 + rLV-Sirt1 group compared with the rLV group (Fig. 9D). Alisertib was used to induce cellular DNA damage, and Western blotting was used to detect the level of DNA damage marker rH2AX (S139). The results showed that the rH2AX(S139) level was significantly reduced in the rLV-miR-26a-1 group compared with the rLV group and increased in the rLV-Cas9-miR-26a1 group compared with the rLV-Cas9 group (Fig. 9E). However, it was not significantly altered in the rLVmiR-26a-1 + rLV-Sirt1 group compared with the rLV group (Fig. 9F). The mismatched DNA plasmid was transfected, and then PCR combined with BamHI-EcoRI restriction analysis was used to detect the level of DNA damage repair. The results showed that the DNA damage repair was significantly increased in the rLV-miR-26a-1 group compared with the rLV group and decreased in the rLV-Cas9-miR-26a-1 group compared with the rLV-Cas9 group (Fig. 9G). After Alisertib induces cellular DNA damage, the DNA damage repair level as significantly increased in the rLV-miR-26a-1 group compared with the rLV group and decreased in the rLV-Cas9-miR-26a-1 group compared with the rLV-Cas9 group (Fig. 9H). DNA microsatellite instability was significantly reducedas significantly increased in the rLV-miR-26a-1 group compared with the rLV group and increased in the rLV-Cas9-miR-26a-1 group compared with the rLV-Cas9 group (Fig. 9l). However, it was not significantly altered in the rLV-miR-26a-1 + rLV-KDM5A group and rLVmiR-26a-1 + rLV-Sirt1 group compared with the rLV group (Fig. 8J). The level of rH2AX (S139) was significantly reduced in the rLV-miR-26a-1 group compared with the rLV group $(31.52 \pm 2.69 \%$ vs $11.93 \pm$ $1.58 \%, P=0.0026<0.01$ ) and increased in the rLV-Cas9-miR-26a-1 group compared with the rLV-Cas9 
group $(34.01 \pm 6.66 \%$ vs $77.04 \pm 7.07 \%, P=0.000122<0.01)$ (Fig. 9K). However, it was not significantly altered in the rLV-miR-26a-1 + rLV-KDM5A group and the rLV-miR-26a-1 + rLV-Sirt1 groupompared with the rLV group $(40.67 \pm 1.06 \%$ vs $43.93 \pm 6.61 \%, P=0.23545>0.05 ; 40.67 \pm 1.06 \%$ vs $37.12 \pm 9.78 \%, P=$ $0.2789>0.05$ ) (Fig. 9L). The degree of DNA damage was significantly reduced in the rLV-miR-26a-1 group compared with the rLV group $(1.65 \pm 0.125$ vs $0.21 \pm 0.091, P=0.0031253<0.01)$ and increased in the rLV-Cas9-miR-26a-1 group compared with the rLV-Cas9 group (1.59 \pm 0.216 vs $3.54 \pm 0.35, P=0.0009218$ < 0.01) (Fig. 9Ma). Next, 8-hydroxy-deoxyguanine (8-OHdG) DNA damage analysis was performed. The results showed that the degree of DNA damage was significantly reduced in the rLV-miR-26a-1 group compared with the rLV group $(0.613 \pm 0.144$ vs 0.233$) \pm 0.115, \mathrm{P}=0.0035<0.01)$ in the rLV-Cas9-miR-26a1 group compared with the rLV-Cas9 group $(0.88 \pm 0.65$ vs $2.07 \pm 0.165, P=0.0 .001524<0.01)$ (Fig. 9Mb).Although the degree of DNA damage was significantly reduced in the rLV-miR-26a-1 group compared with the rLV group $(2.63 \pm 0.411$ vs $0.45 \pm 0.061, P=0.00762<0.01)$. However, it was not significantly altered in the rLV-miR-26a-1 + rLV-KDM5A group and rLV-miR-26a-1 + rLV-Sirt1 group compared with the rLV grou ( $2.63 \pm 0.411$ vs $2.26 \pm 0.69, P=0.3112>0.05 ; 2.63 \pm 0.411$ vs $2.27 \pm 0.806, P$ $=0.29134>0.05)$ (Fig. 9Na). Then, 8-hydroxy-deoxyguanine (8-OHdG) DNA damage analysis was performed, and the results showed that although DNA damage was significantly reduced in the rLV-miR$26 a-1$ group compared with the rLV group $(0.823 \pm 0.061$ vs $0.427 \pm 0.0404, P=0.008298<0.01)$. However, it was not significantly altered in the rLV-miR-26a-1 + rLV-KDM5A group and rLV-miR-26a- $1+$ rLVSirt1 group compared with the rLV group $(0.823 \pm 0.061$ vs $0.86 \pm 0.07, P=0.18403>0.05 ; 0.823 \pm 0.061$ vs $0.746 \pm 0.1242, P=0.230065>0.05$ ) (Fig. $9 \mathrm{Nb}$ ). Collectively, these results suggest that miR-26a-1 promotes the DNA damage repair by inhibiting Sirt1 and KDM5A. [Also see and Supplemental Resuts: miR-26a-1 promotes the DNA damage repair by inhibiting Sirt1 and KDM5A (FigureS10A-N)]

\section{miR-26a-1 triggers the renewal of histones after the repair of DNA damage in liver cancer stem cells}

Given that miR-26a-1 promotes the repair of DNA damage, we will analyze whether miR-26a-1 affects the renewal of histones after the repair of DNA damage in liver cancer stem cells. After DNA damage was induced by alisertib, the binding ability of H3F3A to Skp2, CUL1, and F-box was significantly increased in the rLV-miR-26a-1 group compared with the rLV group and reduced in the rLV-Cas9-miR-26a-1 group compared with the rLV-Cas9 group (Fig. 10A). The binding ability of H3F3A, Skp2, CUL1, and F-box to mismatched DNA damage probes was significantly increased in the rLV-miR-26a-1 group compared with the rLV group and reduced in the rLV-Cas9-miR-26a-1 group compared with the rLV-Cas9 group (Fig. 10B). The H3F3A ubiquitination modification was significantly increased in the rLV-miR-26a-1 group in the rLVmiR-26a-1 group compared with the rLV group and reduced in the rLV-Cas9-miR-26a-1 group compared with the rLV-Cas9 group (Fig. 10C). The ubiquitination level of H3F3A bound by the DNA damage probe was significantly increased in the rLV-miR-26a-1 group compared with the rLV group and reduced in the rLV-Cas9-miR-26a-1 group compared with the rLV-Cas9 group (Fig. 10D). The H3F3A ubiquitination modification was significantly increased in the rLV-miR-26a-1 group compared with the rLV group and reduced in the rLV-Cas9-miR-26a-1 group compared with the rLV-Cas9 group (Fig. 10E). By transfecting mismatched plasmids, the ubiquitination level of H3F3A bound to the DNA damage probe was significantly increased in the rLV-miR-26a-1 group compared with the rLV group and reduced in the rLV- 
Cas9-miR-26a-1 group compared with the rLV-Cas9 group (Fig. 10F). After alisertib was used to induce DNA damage, H3F3A was significantly reduced in in the rLV-miR-26a-1 group compared with the rLV group and increased in the rLV-Cas9-miR-26a-1 group compared with the rLV-Cas9 group (Fig. 10G). The binding ability of H3F3A to the repaired DNA sequence was significantly reduced, and the binding ability of Histone $\mathrm{H} 3$ to the repaired DNA sequence was significantly increased in the rLV-miR-26a-1 group compared with the rLV group and reduced in the rLV-Cas9-miR-26a-1 group compared with the rLV-Cas9 group (Fig. $10 \mathrm{H}$ ). The above results suggest that miR-26a- 1 increases the renewal ability of histones after DNA damage repair, that is, enhances the ability of H3F3A to be replaced by Histone H3. H3F3A was significantly reduced in the rLV-miR-26a-1 group compared with the rLV group. However, it was not significantly altered in the rLV-miR-26a-1 + MG132 group compared with the rLV group (Fig. 10I). The binding ability of H3F3A to the repaired DNA sequence was significantly reduced, and the binding ability of Histone $\mathrm{H} 3$ to the repaired DNA sequence was significantly increased in the rLV-miR-26a-1 group compared with the rLV group. However, it was not significantly altered in the rLV-miR-26a-1 + MG132 group compared with the rLV group (Fig. 10J). Collectively, these results suggest that miR-26a-1 increases the renewal ability of histones dependent on protein ubiquitination degradation pathway after DNA damage repair[Also see and Supplemental Resuts: miR-26a-1 increases the renewal ability of histones dependent on protein ubiquitination degradation pathway after DNA damage repair (FigureS11A-N)].

\section{miR-26a-1 inhibits the expression of cyclins and protein kinase dependent on DNA damage repair in liver cancer stem cells}

Given that miR-26a-1 promotes the repair of DNA damage in liver cancer stem cells, we will consider whether miR-26a-1 inhibits the expression of cyclins and protein kinase dependent on DNA damage repair in liver cancer stem cells. The expression of CDK2, CyclinE, CDK4, CyclinD1, CDK6, CDK8, CyclinM2, CDK15, pRB, PCNA, MAP3K2, PGK1 was significantly reduced and the expression of RB, P18, P21/ The expression of WAF1/Cip1 were significantly increased in the rLV-miR-26a-1 group compared with the rLV group. Similarly, the expression of CDK2, CyclinE, CDK4, CyclinD1, CDK6, CDK8, CyclinM2, CDK15, pRB, PCNA, MAP3K2, and PGK1 were significantly increased and the expression of RB, P18, P21/WAF1/Cip1 were significantly reduced in the rLV-Cas9-miR-26a-1 group compared with the rLV-Cas9 group (Fig. 11A). The expressions of CDK2, CyclinE, CDK4, CyclinD1, CDK6, CDK8, CyclinM2, CDK15, pRB, PCNA, MAP3K2, PGK1 were significantly reduced and the expression of RB, P18, P21 The expression of /WAF1/Cip1 were significantly increased in the rLV-Cas9-miR-26a-1 group compared with Rlv group. However, the expression of CDK2, CyclinE, CDK4, CyclinD1, CDK6, CDK8, CyclinM2, CDK15, pRB, PCNA, MAP3K2, PGK1 were not significantly changed in the rLV-miR-26a-1 + Rucaparib (DNA damage repair inhibitor) group compared with the rLV group (Fig. 11B).Collectively, these results suggest that miR-26a-1 affects the expression of cyclin and protein kinase dependent on DNA damage repair in liver cancer stem cells. [Also see and Supplemental Resuts: miR-26a-1 inhibits the expression of cyclins and protein kinase dependent on DNA damage repair in liver cancer stem cells(FigureS12A-B)].

Excessive Sirt1 and KDM5A abolish the oncogenic function of miR-26a-1 
Since miR-26a-1 promotes the DNA damage repair dependent on Sirt1 and KDM5A and inhibit the growth of liver cancer stem cells in vivo and in vitro, we will analyze whether excessive Sirt1 and KDM5A can abolish the ability of miR-26a- 1 to inhibit the growth of liver cancer stem cells. miR-26a-1-5p/3p ,miR-26a1 precursor were significantly increased in the rLV-miR-26a-1 group, rLV-miR-26a- $1+$ rLV-Sirt1 group, rLVmiR-26a-1 + rLV-KDM5A group compared with the rLV group, respectively $(P<0.01)$ (Fig. 12A-C). The expression of Sirt1 and KDM5A were significantly reduced in the rLV-miR-26a-1 group compared with the rLV group. The expression of Sirt1 was significantly increased in the rLV-miR-26a-1 + rLV-Sirt1 group and decreased in rLV-miR-26a-1 + rLV-KDM5A group compared with the rLV group. KDM5A expression was significantly reduced in rLV-miR-26a-1 + rLV-Sirt1 group and increased in rLV-miR-26a-1 + rLV-KDM5A group compared with the rLV group (Fig. 12D). The cellulr proliferation ability was significantly decreased in the rLV-miR-26a-1 group compared with the rLV group ( 24 hours: $\mathrm{P}=0.0705<0.01$; 48 hours: $\mathrm{P}=$ $0.0788<0.01)$. However, compared with the rLV group ,it was not significantly altered in rLV-miR-26a-1 + rLV-Sirt1 group and rLV-miR-26a-1 + rLV- KDM5A group ( $P>0.05)$ (Fig. 12E). The BrdU positive rate was significantly reduced in the rLV-miR-26a-1 group compared with the rLV group (36.53 $\pm 4.69 \%$ vs $14.27 \pm$ $2.79 \%, P=0.0099<0.01)$. However, it was not significantly altered in rLV-miR-26a- $1+$ rLV- Sirt1 group $(36.53 \pm 4.69 \%$ vs $32.01 \pm 8.18 \%, P=0.085>0.05)$ and the rLV-miR-26a- $1+r L V-K D M 5 A$ group $(36.53 \pm$ $4.69 \%$ vs $31.672 .11 \%, P=<0.01$ ) (Fig. $12 \mathrm{~F}$ ). The colony formation rate was significantly reduced in the rLV-miR-26a-1 group compared with the rLV group $(62.38 \pm 4.503 \%$ vs $27.92 \pm 3.79 \%, P=0.0094<0.01)$. However, it was not significantly altered in rLV-miR-26a-1 + rLV-Sirt1 group $(62.38 \pm 4.503 \%$ vs $59.41 \pm$ $5.46 \%, P=0.2308>0.05)$ and the rLV-miR-26a- $1+$ rLV-KDM5A group $(62.38 \pm 4.503 \%$ vs $55.73 \pm 7.874 \%$, $P=0.1224>0.05$ ) (Fig. 12G). The sphere formation rate was significantly reduced in the rLV-miR-26a-1 group compared with the rLV group $(83.78 \pm 7.05 \%$ vs $37.99 \pm 4.62 \%, P=0.00074<0.01)$. However, it was not significantly altered in rLV-miR-26a- $1+$ rLV-Sirt1 group $(83.78 \pm 7.05 \%$ vs $80.08 \pm 8.95 \%, P=0.3601>$ $0.05)$ and the rLV-miR-26a- $1+r L V-K D M 5 A$ group $(83.78 \pm 7.05 \%$ vs $80.08 \pm 8.95 \%, P=0.30768>0.05)$ (Fig. 12H). The average weight of transplanted tumors was significantly reduced in the rLV-miR-26a-1 group compared with the rLV group $(0.92 \pm 0.12 \mathrm{~g}$ vs $0.32 \pm 0.069 \mathrm{~g}, \mathrm{P}=0.0000000074<0.01)$. However, it was not significantly altered in rLV-miR-26a- $1+r L V$ - Sirt1 group $(0.92 \pm 0.12 \mathrm{~g}$ vs $0.81 \pm 0.16 \mathrm{~g}, \mathrm{P}=0.0732>$ $0.05)$ and rLV-miR-26a-1 + rLV-KDM5A group $(0.92 \pm 0.12 \mathrm{~g}$ vs $0.79 \pm 0.0 .23 \mathrm{~g}, \mathrm{P}=0.062>0.05)$ (Fig. 12l-J). The average xenograft appearance time was significantly increased in the rLV-miR-26a-1 group compared with the rLV group $(8.19 \pm 1.401$ days vs $13.27 \pm 3.47$ days, $P=0.000123<0.01)$. However, it was not significantly altered in rLV-miR-26a-1 + rLV-Sirt1 group ( $8.19 \pm 1.401$ days vs $8.36 \pm 1.43$ days, $\mathrm{P}=$ $0.393975>0.05)$ and the rLV-miR-26a- $1+$ rLV-KDM5A group ( $8.19 \pm 1.401$ days $)$ vs $8.72 \pm 2.57$ days, $\mathrm{P}=$ $0.2466>0.05$ ) (Fig. 12K). The poorly differentiated tumor cells was significantly reduced in the rLV-miR26a-1 group compared with the rLV group . However, it was not significantly altered in the rLV-miR-26a-1 + rLV-Sirt1 group and rLV-miR-26a-1 + rLV-KDM5A group (Fig. 12L). The positive rate of PCNA was significantly reduced in the rLV-miR-26a-1 group compared with the rLV group $(65.19 \pm 11.89 \%$ vs $65.19 \pm$ $11.89 \%, P=0.0000001068<0.01)$ However, it was not significantly altered in the rLV-miR-26a- $1+r L V-$ Sirt1 group $(65.19 \pm 11.89 \%$ vs $59.94 \pm 10.22 \%, P=0.1015>0.05)$ and in the rLV-miR-26a- $1+$ rLV-KDM5A group $(65.19 \pm 11.89 \%$ vs. $62.203 \pm 7.812 \%, P=0.207>0.05)$ (Fig. $12 \mathrm{M} \& N)$. Collectively, these results suggest that Sirt and KDM5A overdose abolish the ability of miR-26-1 to reduce the growth of liver cancer 
stem cells. [Also see and Supplemental Resuts: Excessive Sirt1 and KDM5A abolish miR-26a-1's ability to inhibit the growth of liver cancer stem cells (FigureS13A-N)].

\section{Discussion}

In this study, our results suggest that miR-26a-1 inhibits the NAD(+)-dependent deacetylase Sirt1 expression by targeting the 3 ' non-coding region of Sirt1 which enhances the acetylation modification of histone $\mathrm{H} 4$ on the 16th lysine and the expression of protein arginine methyltransferase PRMT6. Therefore, miR-26a-1 promotes arginine methylation modification of POLB (R137) and Histone. On the other hand, miR-26a-1 inhibits the expression of KDM5A by targeting its 3' non-coding region, which enhances the methylation modification of histone H3 ysine 4. Moreover, miR-26a-1 enhances the expression of histone methyltransferase SETD2 dependent on H3K4me3 and further increases the trimethylation modification of the histone $\mathrm{H} 3$ lysine 36 . Significantly, miR-26a-1 promotes the formation of DNA damage repair complex (Rad51-PARP1-ATR-ATM-hMSH6-XRCC-POLB-SKP2) via H3K36me3. In particular, it was found that miR-26a-1 inhibited the function of long non-coding RNA HULC and promoted the formation of DNA damage repair complex. Furthermore, miR-26a-1 promotes the DNA damage repair ability by promoting the DNA damage repair complex to bind to the DNA damage site, thereby inhibiting the DNA damage of liver cancer stem cells. In particular, miR-26a-1 enhanced the binding of H3F3A to Skp2, CUL1, and F-box at the DNA damage site and enhanced the protein ubiquitination modification of H3F3A, which promoted the Histone $\mathrm{H} 3$ replacng H3F3A, realizing the renewal of histones after DNA damage repair. It was further found that miR-26a-1 inhibited the formation and instability of DNA microsatellites by promoting DNA damage repair, thereby affecting the expression of several cyclins and protein kinases in liver cancer stem cells, such as, inhibiting CDK2 and CyclinE, CDK4, CyclinD1, CDK6, CDK8, CyclinM2, CDK15, pRB, PCNA, MAP3K2, PGK1 and promoting RB, P18, P21/WAF1/Cip1, and thus inhibited the growth of liver cancer stem cells in vivo and in vitro (Fig. 13).

Notably, our results suggest that miR-26a-1 inhibits the growth of human liver cancer stem cells, and excessive Sirt and KDM5A abrogated the oncogenic functions of miR-26-1.Moreover, our results suggest miR-26a-1 targets NAD+-dependent deacetylase Sirt1 and enhances the acetylation modification of histone H4 lysine 16.Sirtuin-1 (SIRT1) is a class-III histone deacetylase (HDAC), an NAD+-dependent enzyme deeply involved in gene regulation, genome stability maintenance, apoptosis, autophagy, senescence, proliferation and tumorigenesis. It also has a key role in the epigenetic regulation of tissue homeostasis and many diseases by deacetylating both histone and non-histone target (11-13). Accumulating evidence has indicated that SIRT1 is a key regulator of DNA damage and cancer $(14,15)$.

Intriguingly, our results suggest that miR-26a-1 enhances the expression of protein arginine methyltransferase PRMT6 gene dependente on H4K16Ac.Protein methyltransferase 6 (PRMT6) to be frequently downregulated in hepatocellular carcinoma (HCC) and regulates RAS/RAF through CRAF methylation(16). PPARa protects against colon carcinogenesis via regulation of PRMT6(17). PTEN arginine methylation by PRMT6 suppresses PI3K-AKT signaling (18). CRAF methylation by PRMT6 regulates hepatocarcinogenesis via ERK-dependent PKM2 nuclear relocalization and activation(19). 
Furthermore, our results suggest that miR-26a-1 enhances the methylation modification of POLB(R137) and histone arginine through PRMT6. Nuclear DNA repair polymerase, POLB, is located in the mitochondria and plays a significant role in mitochondrial BER, mtDNA integrity and mitochondrial function (20). Genome instability caused by a germline mutation in the human DNA repair gene POLB(21)

Interestingly, our results suggest that miR-26a-1 enhances the methylation modification of histone $\mathrm{H} 3$ lysine 4 by reducing KDM5A.KDM5A acts as a negative regulator of p53 signaling (22). KDM5A bound directly to MPC-1 promoter region and suppressed the expression (23). KDM5A/5B knockdown resulted in lower viability of HL-60 cells (24). KDM5A acts as a critical editor of the cells' "histone code" that is required to recruit DNA repair complexes to DNA breaks(25). HDAC1 negatively regulates selective mitotic chromatin in a KDM5A-dependent manner(26). Amplification of KDM5A is observed in many cancers, including breast cancer, prostate cancer, hepatocellular carcinoma (27). The H3K4 tri-demethylase KDM5A and specific COMPASS/KMT2 H3K4 methyltransferases modulate different TSSG loci through H3K4 methylation states and KDM4A recruitment (28).

The initiation and transduction of DNA damage response (DDR) occur in the context of chromatin. JMJD6 modulates DNA damage response through downregulating H4K16Ac (29). MDM2-MOF-H4K16AC axis contributes to tumorigenesis induced by Notch(30). Trimethylation of histone $\mathrm{H} 3$ at lysine 4 (H3K4me3) breadth is linked to cell identity and transcriptional consistency(31). NEK2 promotes tumor growth of gastric cancer cells via regulating KDM5B/H3K4me3(32). SMYD3 promotes metastasis of ovarian cancer via H3K4 me3 (33).

In particular, our results suggest that miR-26a-1 enhances the expression of histone methyltransferase SETD2 and the trimethylation modification on the 36th lysine of Histone H3 dependent on H3K4me3. Loss of SETD2 (the sole histone H3K36 tri-methyltransferase) promotes K-ras-induced carcinogenesis(34). SETD2 restricts prostate cancer metastasis by integrating EZH2 and AMPK signaling pathways (35). SETD2 regulates osteosarcoma cell growth by suppressing Wnt/ $\beta$-catenin signaling(36). The histone mark H3K36me3 regulates human DNA mismatch repair through its interaction with $\operatorname{MutSa}(37,38)$.

Strikingly, our results suggest that miR-26a- 1 enhances the binding ability of Rad51, PARP1, ATR, ATM, hMSH6, XRCC5, POLB, SKP2 to mismatch DNA damage dependent on H3K36me3, and promotes the formation of DNA damage repair complexes. RAD51 promotes homology-directed repair (HDR), replication fork reversal, and stalled fork protection (39). PARP1 blockade is synthetically lethal in XRCC1 deficient sporadic epithelial ovarian cancers (40). the ATM, ATR, DNA-PK family proteins can be activated immediately upon DNA damage recognition (41). The phosphorylation of hMSH6 is involved in cellular signaling of either DNA mismatch repair or MMR-dependent damage recognition activities(43). The hMsh2-hMsh6 complex acts in concert with monoubiquitinated PCNA and Pol $\eta$ in response to oxidative DNA damage in human cells(44). The XRCC genes results in their roles in DNA repair and genetic stability(45). SKP2 promotes tumorigenesis and radiation tolerance through PDCD4 ubiquitination(46). 
Also, our results suggest that that miR-26a-1 promotes the formation of DNA damage repair complexes dependent on long non-coding RNA HULC in liver cancer stem cells. As an oncogene, HULC promotes tumorigenesis by regulating multiple pathways, such as down-regulation of EEF1E1 (47). LncRNA HULC triggers autophagy via stabilizing Sirt1 (48). Circulating extracellular vesicle-encapsulated HULC is a potential biomarker for human pancreatic cancer(49).

In particular, our results suggest that miR-26a-1 promotes the DNA damage repair by inhibiting Sirt1 and KDM5A. On the other hand, our results suggest that miR-26a-1 increases the renewal ability of histones dependent on protein ubiquitination degradation pathway after DNA damage repair. Distinct H3F3A and H3F3B driver mutations define chondroblastoma and giant cell tumor of bone(50). Absence of H3F3A mutation in a subset of malignant giant cell tumor of bone(51). H3F3A promotes lung cancer cell migration through intronic regulation(52). CUL1 is an essential component of SCF (SKP1-CUL1-F-box protein) E3 ubiquitin ligase complex, and promotes breast cancer metastasis through regulating EZH2 (53). F-box proteins have pivotal roles in multiple cellular processes through ubiquitylation and subsequent degradation of target proteins(54).

Ultimately, our results suggest that miR-26a-1 affects the expression of cyclin and protein kinase dependent on DNA damage repair in liver cancer stem cells. CDK2 positively regulates aerobic glycolysis by suppressing SIRT5 in gastric cancer(55). MAP kinase dependent cyclinE/CDK2 activity promotes DNA replication $(56,57)$. p21CIP1 promotes cancer-initiating cells via activation of Wnt/TCF1/CyclinD1 signaling(58). CDK8 promotes angiogenesis in pancreatic cancer via activation of the CDK8- $\beta$-cateninKLF2 signaling axis(59). Recent studies suggest CNNM2 (cyclin M2) to be part of the long-sought basolateral Mg2 + extruder at the renal distal convoluted tubule (60). PA28a/ $\beta$ promotes breast cancer cell invasion and metastasis via down-regulation of CDK15(61). Retinoblastoma protein (pRB) pathway plays a significant role in the development of most human cancers. Loss of pRB results in deregulated cell proliferation and apoptosis(62). Proliferating cell nuclear antigen (PCNA) is known as a molecular marker for proliferation (63). methylation of MAP3K2 by SMYD3 increases MAP kinase signalling and promotes the formation of Ras-driven carcinomas(64). Phosphoglycerate kinase 1 (PGK1) is an important enzyme in the metabolic glycolysis pathway and the acetylation of PGK1 promotes tumorigenesis(65). p18 blocks reprogramming by targeting Cdk4/6-mediated cell cycle regulation(66). Tumor suppressor p21(Waf1/Cip1) functions as a link from p53 to cell-cycle arrest and DNA repair (67).

In conclusions, miR-26a-1 inhibits the expression of NAD+-dependent deacetylase Sirt1 and KDM5A, thereafter, miR-26a-1 promotes DNA damage repair, thereby affecting the expression of some cyclins and protein kinases in liver cancer stem cells. Moreover, excessive Sirt1 and KDM5A abolished miR-26a-1's ability to inhibit the growth of liver cancer stem cells. miR26a-1 may acts as the potential biomarker and therapeutic target for liver cancer. We also shed light on the fact that the attenuation of deregulated functioning of miRNA could be a viable approach for cancer treatment.

\section{Conclusions}


miR-26a-1 inhibits the expression of NAD+-dependent deacetylase Sirt1 and KDM5A, thereafter, miR-26a1 promotes DNA damage repair, thereby affecting the expression of some cyclins and protein kinases in liver cancer stem cells. Moreover, excessive Sirt1 and KDM5A abolished miR-26a-1's ability to inhibit the growth of liver cancer stem cells. miR26a-1 may acts as the potential biomarker and therapeutic target for liver cancer. We also shed light on the fact that the attenuation of deregulated functioning of miRNA could be a viable approach for cancer treatment.

\section{List Of Abbreviations}

RNA G-quadruplexes (RG4s)

sodium dodecyl sulfate-polyacrylamide gel electrophoresis (SDS-PAGE)

RNA Immunoprecipitation(RIP)

3'-uncoding region (UTR)

class-III histone deacetylase (HDAC)

Protein methyltransferase 6 (PRMT6)

\section{Declarations}

\section{Ethics approval and consent to participate}

All methods were carried out in "accordance" with the approved guidelines. All experimental protocols "were approved by" a Tongji university institutional committee. Informed consent was obtained from all subjects. The study was reviewed and approved by the China national institutional animal care and use committee.

\section{Consent for publication}

'Not applicable'

\section{Availability of data and material}

'Not applicable'

\section{Competing interests}

"The authors declare that they have no competing interests"

\section{Authors' contributions}


Dongdong Lu conceived the study and participated in the study design, performance, coordination and manuscript writing. Liyan Wang, Xiaonan Li, Rushi Qin, Yanan Lu, Shuting Song, Yingjie Chen, Sijie Xie, Xiaoxue Jiang performed the research. All authors have read and approved the final manuscript.

\section{Acknowledgements}

This study was supported by grants from National Natural Science Fundation of China (NCSF No.8127229181773158 $₫$ NCSF No.82073130) and by grants from Science and Technology Commission of Shanghai Municipality Shanghai Science and Technology Plan Basic Research Field Project 区20JC1411400खand by grants from Science and Technology Commission of Shanghai Municipality Shanghai Science and Technology Plan Basic Research Field Project囚19JC1415200囚.

\section{References}

1. Acharya A, Berry DC, Zhang H, Jiang Y, Jones BT, Hammer RE, Graff JM, Mendell JT. miR-26 suppresses adipocyte progenitor differentiation and fat production by targeting Fbxl19.Genes Dev. 2019;33(19-20):1367-1380

2. RNA G-quadruplex regulates microRNA-26a biogenesis and function.Liu G, Du W, Xu H, Sun Q, Tang D, Zou S, Zhang Y, Ma M, Zhang G, Du X, Ju S, Cheng W, Tian Y, Fu X. RNA G-quadruplex regulates microRNA-26a biogenesis and function.J Hepatol. 2020;73(2):371-382

3. Peng J, He X, Zhang L, Liu P. MicroRNA-26a protects vascular smooth muscle cells against $\mathrm{H} 2 \mathrm{O} 2$-induced injury through activation of the PTEN/AKT/mTOR pathway.Int J Mol Med. 2018 Sep;42(3):1367-1378

4. Sun YF, Kan Q, Yang Y, Zhang YH, Shen JX, Zhang C, Zhou XY. Knockout of microRNA-26a promotes lung development and pulmonary surfactant synthesis.Mol Med Rep. 2018 Apr;17(4):5988-5995

5. Sekimoto N, Suzuki A, Suzuki Y, Sugano S. Expression of miR-26a exhibits a negative correlation with HMGA1 and regulates cancer progression by targeting HMGA1 in lung adenocarcinoma cells.Mol Med Rep. 2017;15(2):534-542

6. DNA damage and the balance between survival and death in cancer biology. Roos WP, Thomas AD, Kaina B. NA damage and the balance between survival and death in cancer biology.Nat Rev Cancer. 2016 Jan;16(1):20-33

7. Chatterjee N, Walker GC. Mechanisms of DNA damage, repair, and mutagenesis.Environ Mol Mutagen. 2017;58(5):235-263

8. Uckelmann M, Sixma TK. Histone ubiquitination in the DNA damage response.DNA Repair (Amst). 2017; $56: 92-101$

9. Hosoya N, Miyagawa K. Targeting DNA damage response in cancer therapy.Cancer Sci. 2014;105(4):370-88

10. Jin B, Robertson KD. DNA methyltransferases, DNA damage repair, and cancer.Adv Exp Med Biol. 2013;754:3-29. 
11. The Role of SIRT1 on DNA Damage Response and Epigenetic Alterations in Cancer.Alves-Fernandes DK, Jasiulionis MG. The Role of SIRT1 on DNA Damage Response and Epigenetic Alterations in Cancer.Int J Mol Sci. 2019 J;20(13):3153

12. Imperatore F, Maurizio J, Vargas Aguilar S, Busch CJ, Favret J, Kowenz-Leutz E, Cathou W, Gentek R, Perrin P, Leutz A, Berruyer C, Sieweke MH. SIRT1 regulates macrophage self-renewal.EMBO J. 2017;36(16):2353-2372

13. Karbasforooshan $\mathrm{H}$, Roohbakhsh A, Karimi G. SIRT1 and microRNAs: The role in breast, lung and prostate cancers. Exp Cell Res. 2018;367(1):1-6

14. Yang H, Bi Y, Xue L, Wang J, Lu Y, Zhang Z, Chen X, Chu Y, Yang R, Wang R, Liu G. Multifaceted Modulation of SIRT1 in Cancer and Inflammation.Crit Rev Oncog. 2015;20(1-2):49-64

15. Zhao B, Li X, Zhou L, Wang Y, Shang P. SIRT1: a potential tumour biomarker and therapeutic target.J Drug Target. 2019;27(10):1046-1052

16. Chan LH, Zhou L, Ng KY, Wong TL, Lee TK, Sharma R, Loong JH, Ching YP, Yuan YF, Xie D, Lo CM, Man K, Artegiani B, Clevers H, Yan HH, Leung SY, Richard S, Guan XY, Huen MSY, Ma S. PRMT6 Regulates RAS/RAF Binding and MEK/ERK-Mediated Cancer Stemness Activities in Hepatocellular Carcinoma through CRAF Methylation.Cell Rep. 2018;25(3):690-701

17. Luo Y, Xie C, Brocker CN, Fan J, Wu X, Feng L, Wang Q, Zhao J, Lu D, Tandon M, Cam M, Krausz KW, Liu W, Gonzalez FJ. Intestinal PPARa Protects Against Colon Carcinogenesis via Regulation of Methyltransferases DNMT1 and PRMT6.Gastroenterology. 2019;157(3):744-759

18. Feng J, Dang Y, Zhang W, Zhao X, Zhang C, Hou Z, Jin Y, McNutt MA, Marks AR, Yin Y. PTEN arginine methylation by PRMT6 suppresses PI3K-AKT signaling and modulates pre-mRNA splicing.Proc Natl Acad Sci U S A. 2019;116(14):6868-6877

19. Wong TL, Ng KY, Tan KV, Chan LH, Zhou L, Che N, Hoo RLC, Lee TK, Richard S, Lo CM, Man K, Khong PL, Ma S. CRAF Methylation by PRMT6 Regulates Aerobic Glycolysis-Driven Hepatocarcinogenesis via ERK-Dependent PKM2 Nuclear Relocalization and Activation. Hepatology. 2020;71(4):1279-1296

20. Kaufman BA, Van Houten B. POLB: A new role of DNA polymerase beta in mitochondrial base excision repair.DNA Repair (Amst). 2017 Dec;60:A1-A5

21. Sobol RW. Genome instability caused by a germline mutation in the human DNA repair gene POLB.PLoS Genet. 2012;8(11):e1003086

22. KDM5Aregulates a translational program that controls $p 53$ protein expression. Hu D, Jablonowski $C$, Cheng PH, AlTahan A, Li C, Wang Y, Palmer L, Lan C, Sun B, Abu-Zaid A, Fan Y, Brimble M, Gamboa NT, Kumbhar RC, Yanishevski D, Miller KM, Kang G, Zambetti GP, Chen T, Yan Q, Davidoff AM, Yang J. KDM5ARegulates a Translational Program that Controls p53 Protein Expression.iScience. 2018;9:84100

23. Cui J, Quan M, Xie D, Gao Y, Guha S, Fallon MB, Chen J, Xie K. A novel KDM5A/MPC-1 signaling pathway promotes pancreatic cancer progression via redirecting mitochondrial pyruvate metabolism.Oncogene. 2020 Jan;39(5):1140-1151 
24. Shokri G, Doudi S, Fathi-Roudsari M, Kouhkan F, Sanati MH. Targeting histone demethylasesKDM5Aand KDM5B in AML cancer cells: A comparative view.Leuk Res. 2018;68:105111

25. Price BD. KDM5A demethylase: Erasing histone modifications to promote repair of DNA breaks.J Cell Biol. 2017 Jul 3;216(7):1871-1873

26. Dreval K, Lake RJ, Fan HY. HDAC1 negatively regulates selective mitotic chromatin binding of the Notch effector RBPJ in a KDM5A-dependent manner.Nucleic Acids Res. 2019;47(9):4521-4538

27. Petronikolou N, Longbotham JE, Fujimori DG. Extended Recognition of the Histone H3 Tail by Histone DemethylaseKDM5A.Biochemistry. 2020;59(5):647-651

28. Mishra S, Van Rechem C, Pal S, Clarke TL, Chakraborty D, Mahan SD, Black JC, Murphy SE, Lawrence MS, Daniels DL, Whetstine JR. Cross-talk between Lysine-Modifying Enzymes Controls Site-Specific DNA Amplifications.Cell. 2018;174(4):803-817.e16

29. Huo D, Chen H, Cheng Y, Song X, Zhang K, Li MJ, Xuan C. JMJD6 modulates DNA damage response through downregulating H4K16acindependently of its enzymatic activity.Cell Death Differ. 2020;27(3):1052-1066

30. Liu Y, Xing ZB, Wang SQ, Chen S, Liu YK, Li YH, Li YF, Wang YQ, Lu Y, Hu WN, Zhang JH. MDM2-MOFH4K16ac axis contributes to tumorigenesis induced by Notch.FEBS J. 2014;281(15):3315-24

31. Benayoun BA, Pollina EA, Ucar D, Mahmoudi S, Karra K, Wong ED, Devarajan K, Daugherty AC, Kundaje AB, Mancini E, Hitz BC, Gupta R, Rando TA, Baker JC, Snyder MP, Cherry JM, Brunet A. H3K4me3 breadth is linked to cell identity and transcriptional consistency.Cell. 2014;158(3):673-88

32. Li Y, Chen L, Feng L, Zhu M, Shen Q, Fang Y, Liu X, Zhang X. NEK2 promotes proliferation, migration and tumor growth of gastric cancercells via regulating KDM5B/H3K4me3.Am J Cancer Res. 2019;9(11):2364-2378

33. Lyu T, Jiang Y, Jia N, Che X, Li Q, Yu Y, Hua K, Bast RC Jr, Feng W. SMYD3 promotes implant metastasis of ovarian cancer via H3K4 trimethylation of integrin promoters.Int $\mathrm{J}$ Cancer. 2020;146(6):1553-1567

34. Niu N, Lu P, Yang Y, He R, Zhang L, Shi J, Wu J, Yang M, Zhang ZG, Wang LW, Gao WQ, Habtezion A, Xiao GG, Sun Y, Li L, Xue J.Gut. 2020;69(4):715-726

35. Yuan H, Han Y, Wang X, Li N, Liu Q, Yin Y, Wang H, Pan L, Li L, Song K, Qiu T, Pan Q, Chen Q, Zhang G, Zang Y, Tan M, Zhang J, Li Q, Wang X, Jiang J, Qin J. SETD2 Restricts Prostate Cancer Metastasis by Integrating EZH2 and AMPK Signaling Pathways.Cancer Cell. 2020:S1535-6108(20)30272-5

36. Jiang C, He C, Wu Z, Li F, Xiao J. Histone methyltransferase SETD2 regulates osteosarcoma cell growth and chemosensitivity by suppressing Wnt/ $\beta$-catenin signaling.Biochem Biophys Res Commun. 2018;502(3):382-388

37. Fang J, Huang Y, Mao G, Yang S, Rennert G, Gu L, Li H, Li GM. Cancer-driving H3G34V/R/D mutations block H3K36 methylation and H3K36me3-MutSa interaction.Proc Natl Acad Sci U S A. 2018 Sep 18;115(38):9598-9603 
38. Li F, Mao G, Tong D, Huang J, Gu L, Yang W, Li GM. The histone mark H3K36me3 regulates human DNA mismatch repair through its interaction with MutSa.Cell. 2013;153(3):590-600

39. Dungrawala $H$, Bhat KP, Le Meur R, Chazin WJ, Ding X, Sharan SK, Wessel SR, Sathe AA, Zhao R, Cortez D. RADX Promotes Genome Stability and Modulates Chemosensitivity by

Regulating RAD51 at Replication Forks.Mol Cell. 2017;67(3):374-386

40. Ali R, Alabdullah M, Alblihy A, Miligy I, Mesquita KA, Chan SY, Moseley P, Rakha EA, Madhusudan S. PARP1 blockade is synthetically lethal in XRCC1 deficient sporadic epithelial ovarian cancers.Cancer Lett. 2020;469:124-133

41. Ma M, Rodriguez A, Sugimoto K. Activation of ATR-related protein kinase upon DNA damage recognition.Curr Genet. 2020 Apr;66(2):327-333

42. Weber AM, Ryan AJ. ATM and ATR as therapeutic targets in cancer.Pharmacol Ther. 2015;149:124-38

43. Kaliyaperumal S, Patrick SM, Williams KJ.Phosphorylated hMSH6: DNA mismatch versus DNA damage recognition.Mutat Res. 2011;706(1-2):36-45T

44. Zlatanou A, Despras E, Braz-Petta T, Boubakour-Azzouz I, Pouvelle C, Stewart GS, Nakajima S, Yasui A, Ishchenko AA, Kannouche PL. The hMsh2-hMsh6 complex acts in concert with monoubiquitinated PCNA and Pol $\eta$ in response to oxidative DNA damage in human cells.Mol Cell. 2011;43(4):649-62

45. Thacker J, Zdzienicka MZ. The mammalian XRCC genes: their roles in DNA repair and genetic stability.DNA Repair (Amst). 2003;2(6):655-72

46. Li C, Du L, Ren Y, Liu X, Jiao Q, Cui D, Wen M, Wang C, Wei G, Wang Y, Ji A, Wang Q. SKP2 promotes breast cancer tumorigenesis and radiation tolerance through PDCD4 ubiquitination.J Exp Clin Cancer Res. 2019;38(1):76.

47. Yu X, Zheng H, Chan MT, Wu WK. HULC: an oncogenic long non-coding RNA in human cancer.J Cell Mol Med. 2017 Feb;21(2):410-417

48. Xiong H, Ni Z, He J, Jiang S, Li X, He J, Gong W, Zheng L, Chen S, Li B, Zhang N, Lyu X, Huang G, Chen $B$, Zhang $Y$, He F. LncRNA HULC triggers autophagy via stabilizing Sirt1 and attenuates the chemosensitivity of HCC cells.Oncogene. 2017;36(25):3528-3540

49. Takahashi K, Ota Y, Kogure T, Suzuki Y, Iwamoto H, Yamakita K, Kitano Y, Fujii S, Haneda M, Patel T, Ota T. Circulating extracellular vesicle-encapsulated HULC is a potential biomarker for human pancreatic cancer.Cancer Sci. 2020 Jan;111(1):98-111.

50. Behjati S, Tarpey PS, Presneau N, Scheipl S, Pillay N, Van Loo P, Wedge DC, Cooke SL, Gundem G, Davies H, Nik-Zainal S, Martin S, McLaren S, Goodie V, Robinson B, Butler A, Teague JW, Halai D, Khatri B, Myklebost O, Baumhoer D, Jundt G, Hamoudi R, Tirabosco R, Amary MF, Futreal PA, Stratton MR, Campbell PJ, Flanagan AM. Distinct H3F3A and H3F3B driver mutations define chondroblastoma and giant cell tumor of bone.Nat Genet. 2013;45(12):1479-82

51. Yoshida KI, Nakano Y, Honda-Kitahara M, Wakai S, Motoi T, Ogura K, Sano N, Shibata T, Okuma T, Iwata S, Kawai A, Ichimura K, Yoshida A. Absence of H3F3A mutation in a subset of malignant giant cell tumor of bone.Mod Pathol. 2019;32(12):1751-1761 
52. Park SM, Choi EY, Bae M, Kim S, Park JB, Yoo H, Choi JK, Kim YJ, Lee SH, Kim IH. Histone variant H3F3A promotes lung cancer cell migration through intronic regulation.Nat Commun. 2016;7:12914

53. Huang YF, Zhang Z, Zhang M, Chen YS, Song J, Hou PF, Yong HM, Zheng JN, Bai J. CUL1 promotes breast cancer metastasis through regulating $\mathrm{EZH}$ 2-induced the autocrine expression of the cytokines CXCL8 and IL11.Cell Death Dis. 2018;10(1):2

54. Wang Z, Liu P, Inuzuka H, Wei W. Roles of F-boxproteins in cancer.Nat Rev Cancer. 2014;14(4):233-47

55. Tang Z, Li L, Tang Y, Xie D, Wu K, Wei W, Xiao Q. CDK2 positively regulates aerobic glycolysis by suppressing SIRT5 in gastric cancer.Cancer Sci. 2018;109(8):2590-2598

56. Kisielewska J, Philipova R, Huang JY, Whitaker M. MAP kinase dependent cyclinE/cdk2 activity promotes DNA replication in early sea urchin embryos.Dev Biol. 2009;334(2):383-94

57. Goel S, DeCristo MJ, McAllister SS, Zhao JJ. CDK4/6 Inhibition inCancer: Beyond Cell Cycle Arrest.Trends Cell Biol. 2018;28(11):911-925

58. Benard O, Qian X, Liang H, Ren Z, Suyama K, Norton L, Hazan RB. p21CIP1 Promotes Mammary Cancer-Initiating Cells via Activation of Wnt/TCF1/CyclinD1 Signaling.Mol Cancer Res.2019;17(7):1571-1581

59. Wei R, Kong L, Xiao Y, Yuan H, Song Y, Wang J, Yu H, Mao S, Xu W. CDK8 regulates the angiogenesis of pancreatic cancer cells in part via the CDK8- $\beta$-catenin-KLF2 signal axis.Exp Cell Res. 2018;369(2):304-315

60. Corral-Rodríguez MÁ, Stuiver M, Abascal-Palacios G, Diercks T, Oyenarte I, Ereño-Orbea J, de Opakua Al, Blanco FJ, Encinar JA, Spiwok V, Terashima H, Accardi A, Müller D, Martínez-Cruz LA. Nucleotide binding triggers a conformational change of the CBS module of the magnesium transporterCNNM2from a twisted towards a flat structure.Biochem J. 2014;464(1):23-34

61. Li S, Dai X, Gong K, Song K, Tai F, Shi J. PA28a/ $\beta$ Promote Breast Cancer Cell Invasion and Metastasis via Down-Regulation of CDK15.Front Oncol. 2019;9:1283

62. Hickman ES, Moroni MC, Helin K. The role of p53 and pRB in apoptosis and cancer.Curr Opin Genet Dev. 2002;12(1):60-6

63. Wang SC. PCNA: a silent housekeeper or a potential therapeutic target?Trends Pharmacol Sci. 2014;35(4):178-86

64. Mazur PK, Reynoird N, Khatri P, Jansen PW, Wilkinson AW, Liu S, Barbash O, Van Aller GS, Huddleston M, Dhanak D, Tummino PJ, Kruger RG, Garcia BA, Butte AJ, Vermeulen M, Sage J, Gozani O. SMYD3 links lysine methylation of MAP3K2 to Ras-driven cancer.Nature. 2014;510(7504):283-7

65. Hu H, Zhu W, Qin J, Chen M, Gong L, Li L, Liu X, Tao Y, Yin H, Zhou H, Zhou L, Ye D, Ye Q, Gao D. Acetylation ofPGK1 promotes liver cancer cell proliferation and tumorigenesis. Hepatology. 2017;65(2):515-528)

66. Zhu S, Cao J, Sun H, Liu K, Li Y, Zhao T. p18inhibits reprogramming through inactivation of Cdk4/6.Sci Rep. 2016;6:31085 
67. Yang W, Qi Q, Zhang H, Xu W, Chen Z, Wang L, Wang Y, Dong X, Jiao H, Huo Z. p21Waf1/Cip1polymorphisms and risk of esophageal cancer.Ann Surg Oncol. 2010;17(5):1453-8)

\section{Figures}
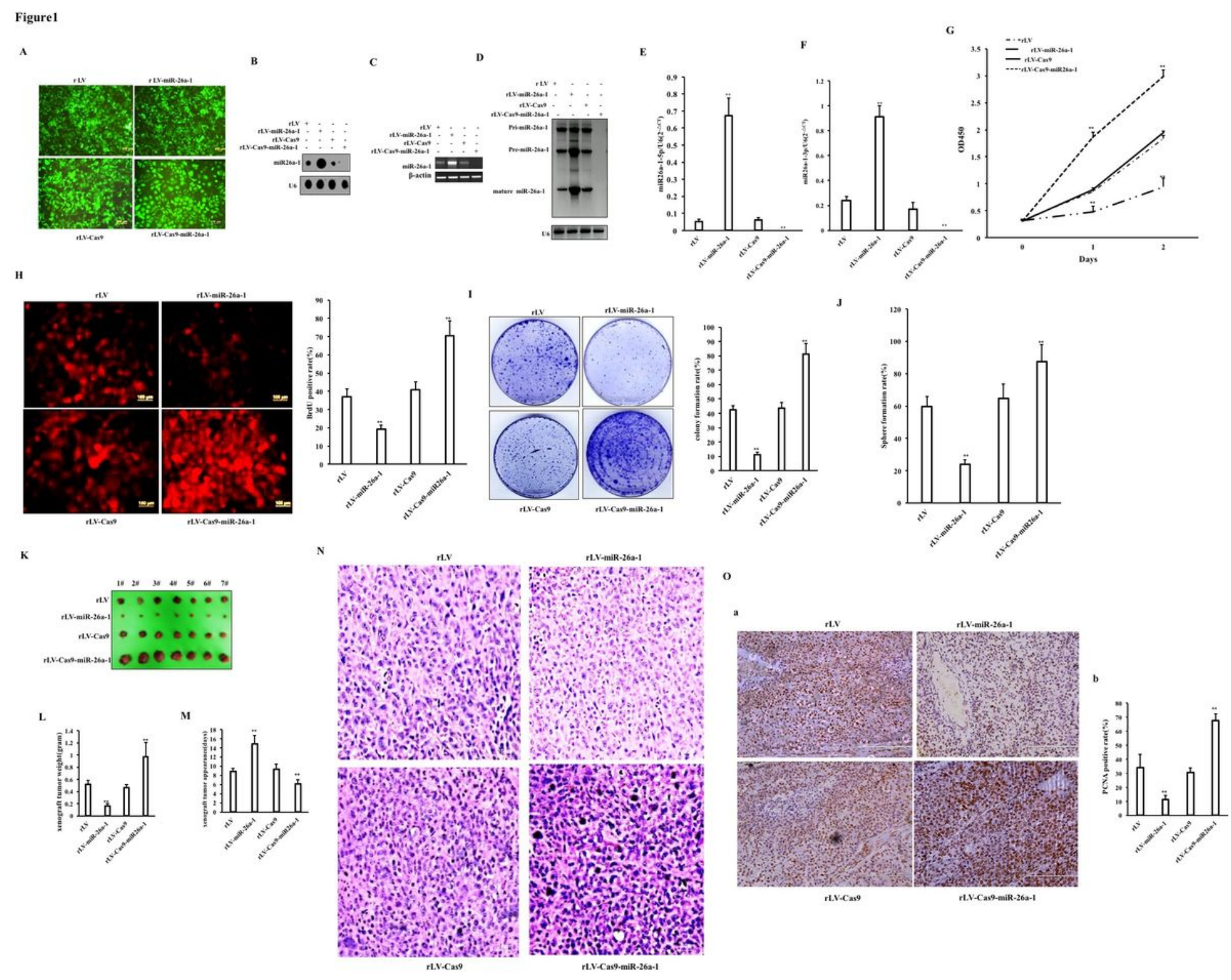

\section{Figure 1}

miR-26a-1 inhibits the growth of human liver cancer stem cells in vitro and in vivo. A. The positive cells were picked under a fluorescent microscope (100 times). B. Dot-Blot was used to detect the expression of miR-26a-1. U6 as the internal reference gene. C. RT-PCR was used to detect the expression of miR-26a-1 precursor . $\beta$-actin as an internal reference gene. D. Northern blotting was used to detect miR-26a-1 . U6 as the internal reference gene. E-F. Real-time RT-PCR was used to detect miR-26a. U6 is used as the internal reference gene. G. growth curve determination (CCK8). $\mathrm{H}$. BrdU staining was used to determine the $\mathrm{S}$ phase in hLCSCs cells. a. BrdU stain. b. the percentage of $S$ phase. I.the plate colony forming ability was 
determined. a. Photographs of colonies.b. colony formation rate. Each group value is expressed as mean \pm standard deviation (bar $\pm S E M, n=3$ ), **, $P<0.01, *, P<0.05$ means the statistical difference is significant. J. The sphere forming ability of hLCSCs cells was measured. K. Liver cancer stem cells were respectively inoculated into the armpits of immunocompromised Balb/C nude mice ( 7 in each group, 28 in total), a photo of xenograft which was dissected 1 month later. L. Comparison of the size (grams) of transplanted tumors in nude mice. M. Comparison of the time (days) for the appearance of transplanted tumors in nude mice. N. Tumor tissue sections $(4 \mu \mathrm{m})$ fixed in $4 \%$ formaldehyde and embedded in paraffin were stained with hematoxylin-eosin (HE) (original magnification $\times 100$ ). 0.a. The transplanted tumor tissue sections $(4 \mu \mathrm{m})$ of nude mice fixed with $4 \%$ formaldehyde and paraffin embedded were subjected to anti-PCNA immunohistochemical staining (original magnification $\times 100$ ). b. comparison of PCNA positive rate.

Figure 2

A

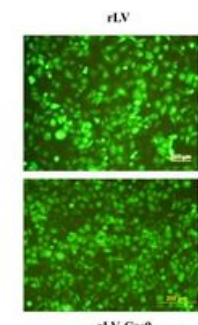

н

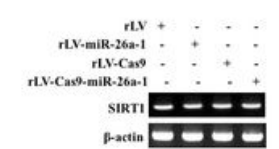

sirTi-
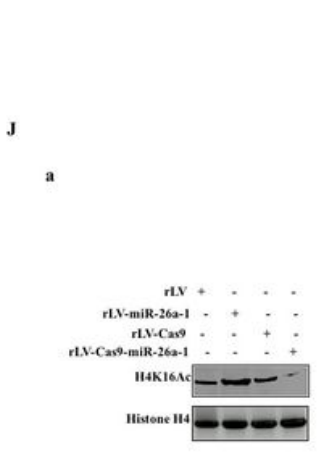

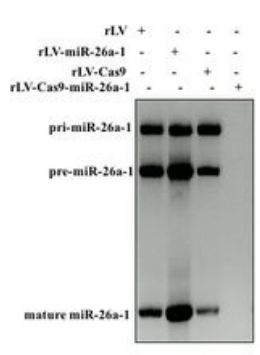

U -50
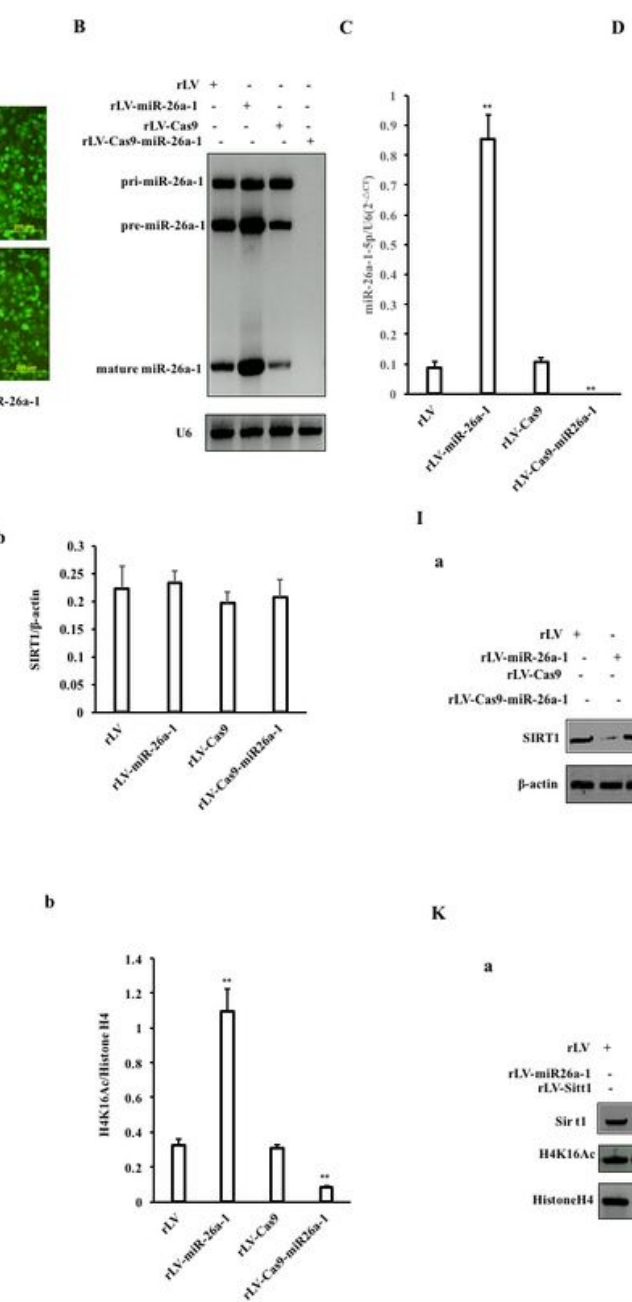

E

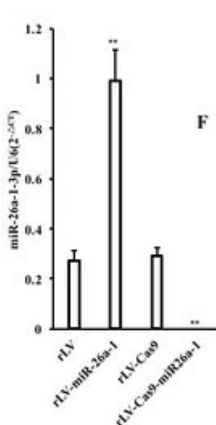

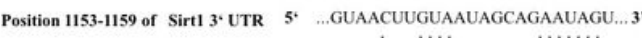
has-miR-26a-1-3p $3^{\prime}$ GCACGUUCAUU-GGUUCUUAUCC-
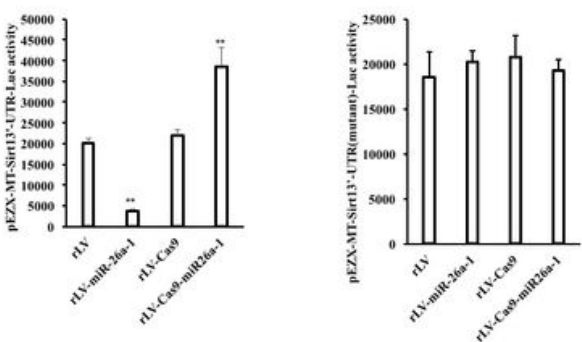

K
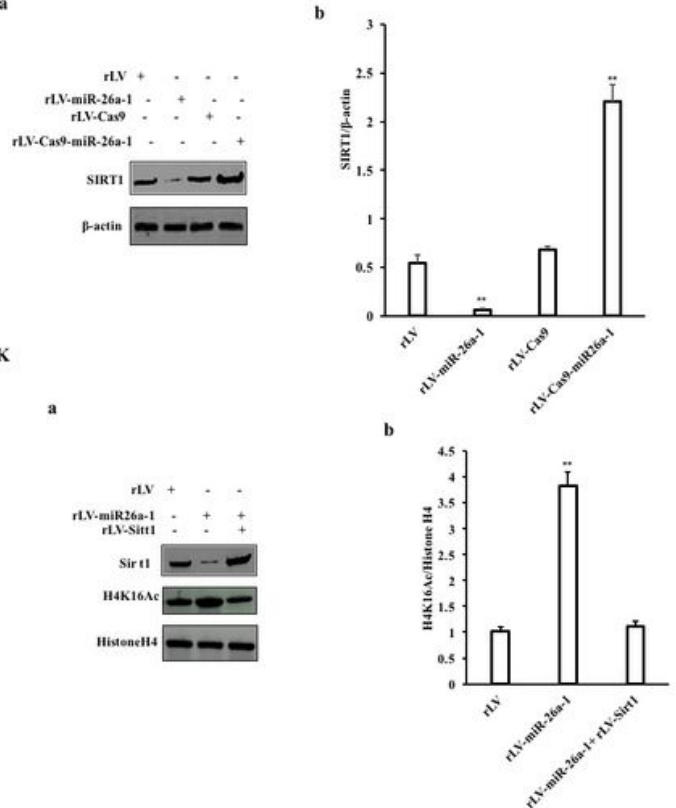

\section{Figure 2}

miR-26a-1 targets NAD+-dependent deacetylase Sirt1 in human liver cancer stem cells and enhances the acetylation modification of histone $\mathrm{H} 4$ lysine 16. A. Image taken with a fluorescence microscope. $\mathrm{B}$. Northern blotting was used to detect the miR-26a-1 in these four stable cell lines, U6 as an internal reference gene. C. quantitative RT-PCR was used to detect miR-26a-1-3p in these four stable cell lines.U6 
was used as internal reference gene. D. Quantitative RT-PCR was used to detect miR-26a-1-5p in these four stable cell lines. U6 was used as internal reference gene. E. MirTarget scanning soft software and BLAST tools was used to analyze the combined seed sequence of mature miR-26a-1 to Sirt1 mRNA 3'uncoding region (UTR). F. pEZX-MT-Sirt1 3'UTR-Luc dual luciferase reporter gene activity. G. pEZX-MTSirt13'UTR (mutant)-Luc dual luciferase reporter gene activity. H. RT-PCR was used to detect the transcriptional ability of Sirt1. $\beta$-actin was used as an internal reference gene. I. Western blotting was used to detect the translation ability of Sirt1. $\beta$-actin was used as an internal reference gene. J. Western blotting was used to detect $\mathrm{H} 4 \mathrm{~K} 6 \mathrm{Ac}$. Histone $\mathrm{H} 4$ was used as the internal reference gene. K. Western blotting was used to detect H4K6Ac. Histone H4 was used as the internal reference gene.

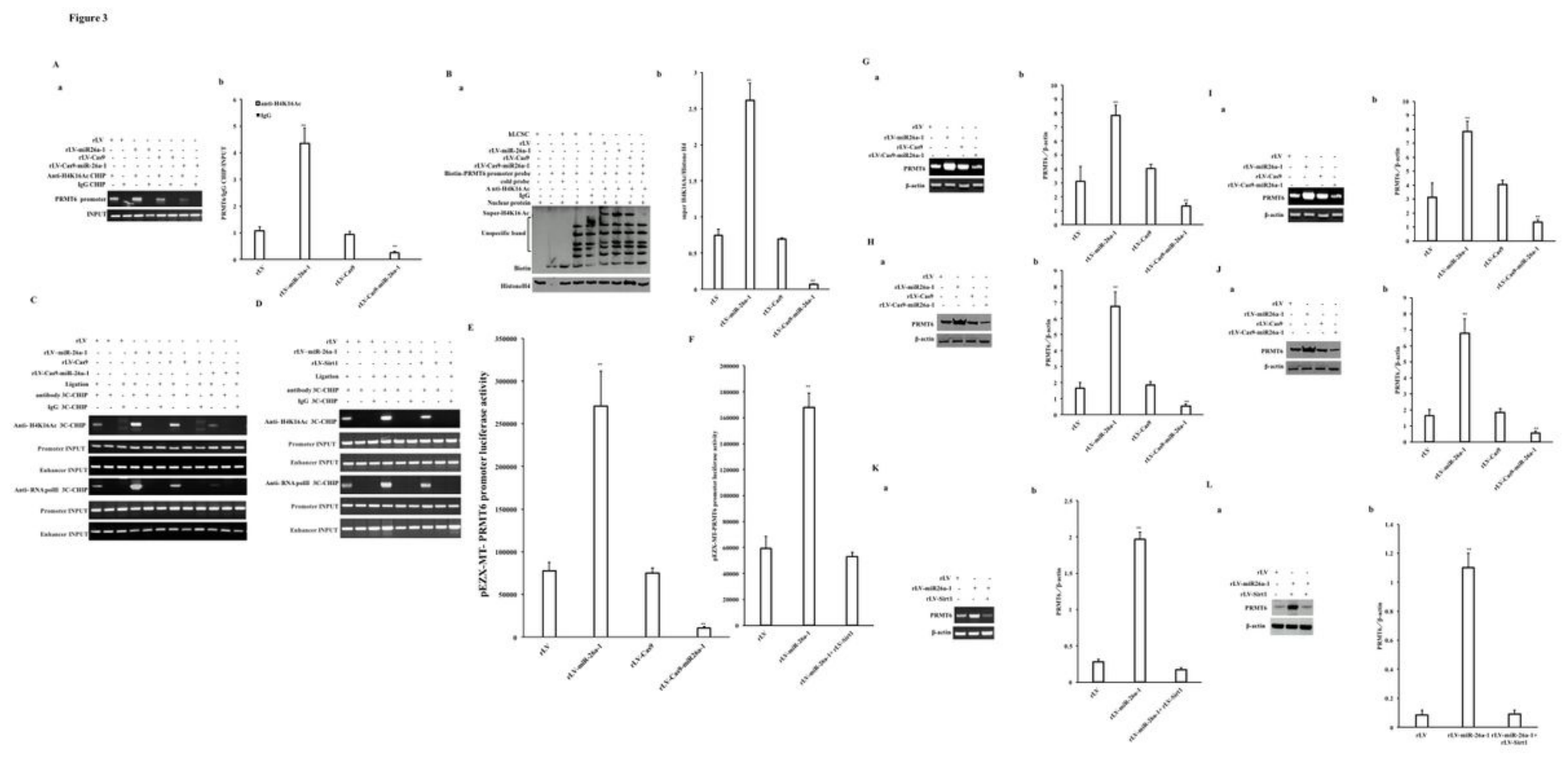

\section{Figure 3}

miR-26a-1 enhances the expression of protein arginine methyltransferase PRMT6. A. The chromosome immunoprecipitation (CHIP) with anti-H4K16Ac. The DNA isolated and purified from the CHIP precipitates was used as a template, and PCR amplification was carried out with primers designed according to the PRMT6 promoter sequence. IgG CHIP was used as a negative control. B. Super-DNA-protein complex gel migration assay (Super-EMSA) with biotin-PRMT6 promoter and anti-H4K16Ac, with IgG super- EMSA was used as a negative control. EMSA without nucleoprotein and EMSA with excessive cold probe were used as system reference, and the hybridization band of Biotin and the amount of nucleoprotein added were used as INPUT. C. Chromosome configuration capture (3C)-chromatin immunoprecipitation (CHIP) using anti-RNApolll and anti-H4K16Ac. The DNA isolated and purified from the CHIP-3C precipitate was used as a template, and a pair of mixed primers designed according to the PRMT6 promoter-enhancer were used for polymerase chain reaction (PCR) amplification. IgG CHIP-3C was used as a negative control; the DNA retained before chromatin immunoprecipitation was used as a template, and the products amplified by independent primers designed by PRMT6 promoter-enhancer were used as internal 
reference (INPUT). D. Chromosome configuration capture (3C)-chromatin immunoprecipitation (CHIP) using anti-RNApolll and anti-H4K16Ac. E-F. The analysis of activity of the pEZX-MT-PRMT6 promoter-Luc reporter gene. G. Reverse transcription polymerase chain reaction (RT-PCR) was used to detect the transcription ability of PRMT6 . $\beta$-actin as an internal reference gene. H. Western blotting was used to detect the translation ability of PRMT6 . $\beta$-actin as an internal reference gene. I. Reverse transcription polymerase chain reaction (RT-PCR) was used to detect the transcription ability of PRMT6. $\beta$-actin as an internal reference gene. $\mathrm{H}$. Western blotting was used to detect the translation ability of PRMT6 . $\beta$-actin as an internal reference gene.

Figure 4

$\mathbf{A}$

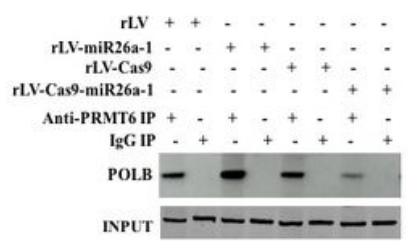

C

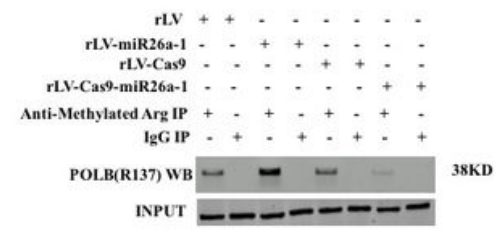

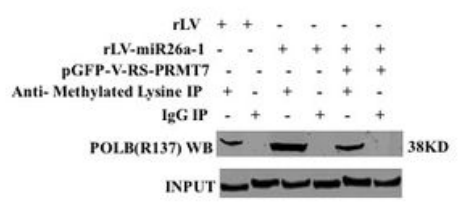

E

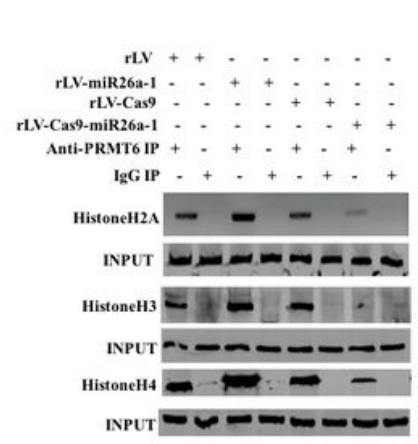

$\mathbf{F}$

G
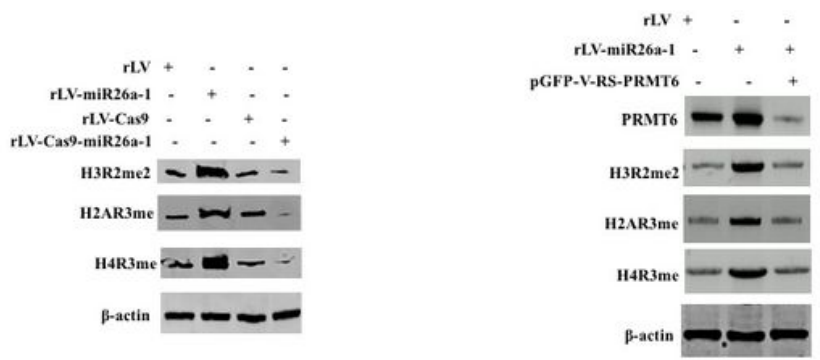

\section{Figure 4}

miR-26a-1 promotes the methylation modification of POLB (R137) and histone arginine through PRMT6. A. Western blotting was used to detect the translation ability of POLB . $\beta$-actin serves as an internal reference gene. B. Co- immunoprecipitation with anti-PRMT6 and Western blotting analysis with antiPOLB. IgG immunoprecipitation was used as a negative control, and the samples before coprecipitation were detected by Western blotting with anti-PRMT6 as INPUT. C-D. Co-immunoprecipitation with antiMethylated Arg and Western blotting analysis with anti-POLB (R137). IgG immunoprecipitation was used as a negative control. E. Co-immunoprecipitation with anti-PRMT6 and then Western blotting with anti$\mathrm{H} 2 \mathrm{~A}$, anti-H3, and anti-H4. IgG immunoprecipitation was used as a negative control, and the samples before coprecipitation were detected by Western blotting with anti-PRMT6 as INPUT. F. Western blotting was used to detect the modification levels of H3R2me2, H2AR3me, and H4R3me. H2A, H3, and H4 as internal reference genes. G. Western blotting was used to detect the modification level of H3R2me2, H2AR3me, H4R3me and the expression of PRMT6. H2A, H3, and H4 as internal reference genes. 
A

Position 4606-4612 of KDM5A 3' UTR 5 ' ...CUAUUAUCAUUgCUGAGAaUAGU...3

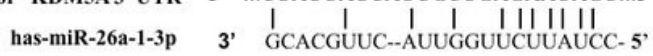
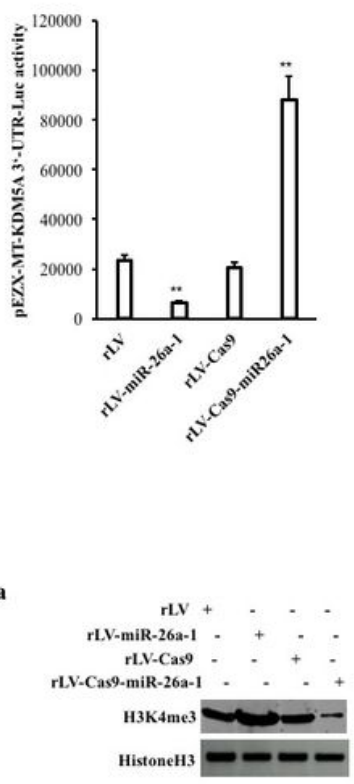

C

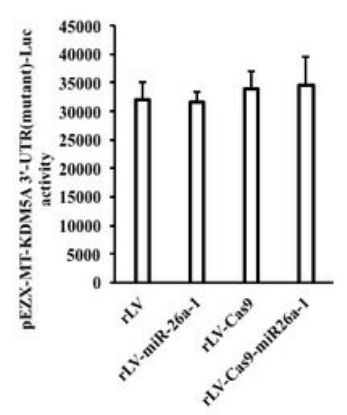

b

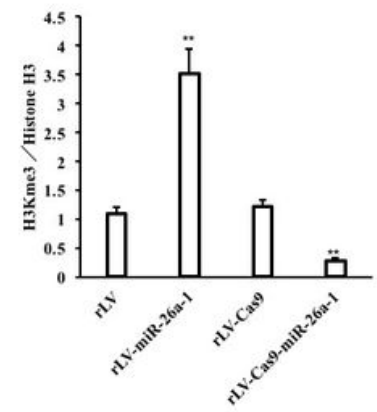

D

a

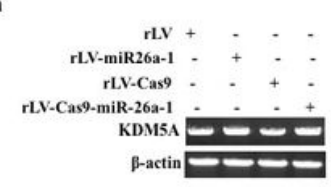

$\mathbf{E}$

a

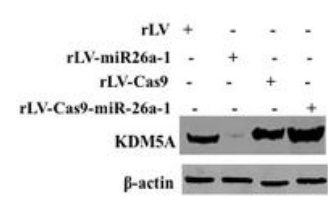

G

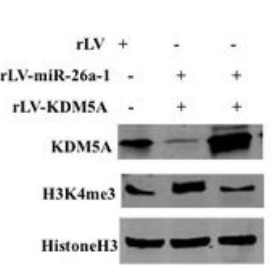

b
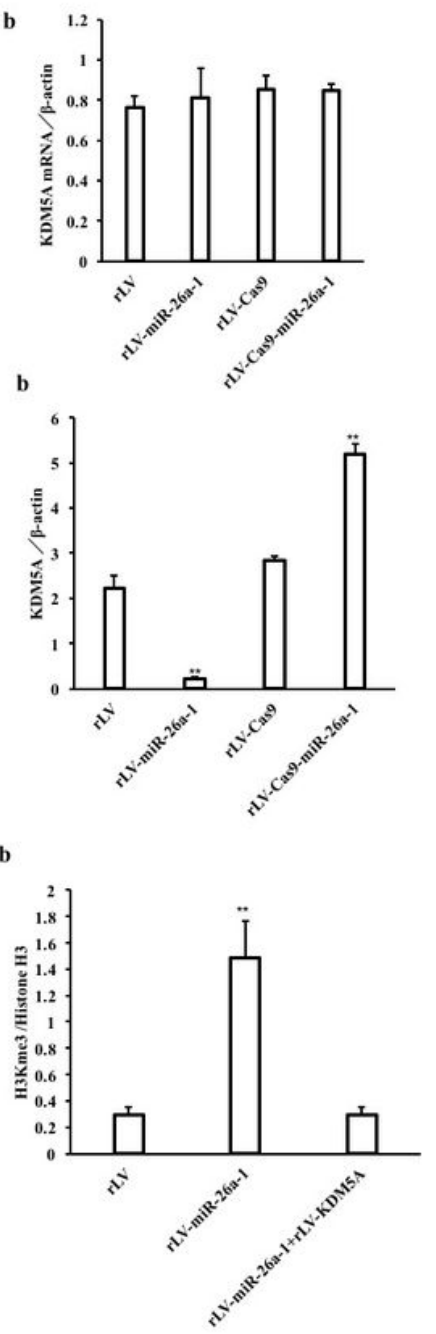

Figure 5

miR-26a-1 enhances the methylation modification of histone $\mathrm{H} 3$ lysine 4 via targeting KDM5A. A. The binding of seed sequence of mature miR-26a-1 to KDM5A mRNA 3'-uncoding region (UTR) was analyzed by MirTarget scanning soft software and BLAST Tools analyze. B. The activity of the pEZX-MT-KDM5A 3'UTR-Luc luciferase reporter gene. Each experiment was repeated three times. Each group value is expressed as mean \pm standard deviation (mean $\pm S E M, n=3$ ), **, $P<0.01, *, P<0.05$. C. The activity of the pEZX-MT-KDM5A 3'UTR(mutant)-Luc luciferase reporter gene was detected. D. RT-PCR was used to detect the transcription ability of KDM5A. $\beta$-actin is used as an internal reference gene. E. Western blotting was used to detect the translation ability of KDM5A. $\beta$-actin as an internal reference gene. F-G. Western blotting was used to detect $\mathrm{H} 3 \mathrm{~K} 4 \mathrm{me} 3$. Histone $\mathrm{H} 3$ was used as an internal reference gene. 


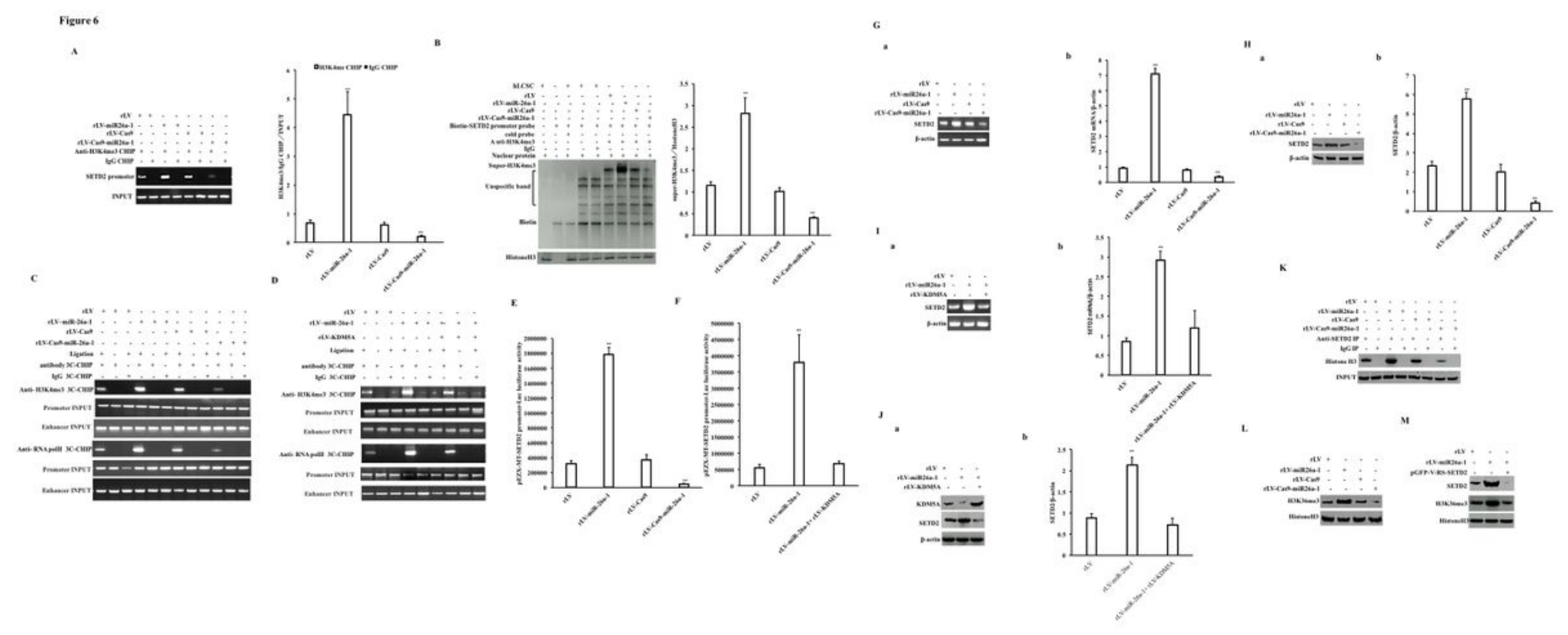

Figure 6

miR-26a-1 enhances the expression of histone methyltransferase SETD2 and the trimethylation modification on the 36th lysine of Histone H3 dependent on H3K4me3. A. Chromosome immunoprecipitation (CHIP) with anti-H3K4me3. The DNA isolated and purified from the CHIP precipitate was used as a template, and the primers designed according to the SETD2 promoter sequence were used for polymerase chain reaction (PCR) amplification. IgG CHIP is used as a negative control. B. SuperDNA gel migration was used to measure the binding ability of histone methyltransferase SETD2 promoter probe and H3K4me3. Super-DNA-protein complex gel migration experiment (Super-EMSA) using Biotin labeled SETD2 promoter probe (Biotin-SETD2 promoter) and anti-H3K4me3, anti-Biotin .IgG super- EMSA was used as a negative contro. C-D. Chromosome configuration capture (3C)-chromatin immunoprecipitation (CHIP) using anti-RNApolll and anti-P300. The DNA isolated and purified from the CHIP-3C precipitate was used as a template, and a pair of mixed primers designed based on the histone methyltransferase SETD2 promoter-enhancer were used for polymerase chain reaction (PCR) amplification. IgG CHIP-3C was used as a negative control; the DNA retained before chromatin immunoprecipitation was used as a template, and the products amplified by independent primers designed with the histone methyltransferase SETD2 promoter-enhancer were used as the internal reference (INPUT). E-F. the activity of the pEZX-MT-SETD2 promoter-Luc luciferase reporter gene. Each experiment was repeated three times. Each group value is expressed as mean \pm standard deviation (mean $\pm S E M, n=3$ ), **, $P<0.01, *, P<0.05$. G. RT-PCR was used to detect the transcription ability of histone methyltransferase SETD2 . $\beta$-actin as an internal reference gene. H. Western blotting is used to detect the translation ability of the histone methyltransferase SETD2. $\beta$-actin as the internal reference gene. I. RTPCR was used to detect the transcription ability of t SETD2 . $\beta$-actin as an internal reference gene. J. Western blotting was used to detect the translation ability of SETD2. $\beta$-actin as an internal reference gene. K. Co-immunoprecipitation with anti-SETD2 and Western blotting analysis with anti-Histone H3. IgG immunoprecipitation was used as a negative control, and the sample before coprecipitation was detected 
by Western blotting with anti-SETD2 as INPUT. L-M. Western blotting was used to detect the H3K36me3.Histone H3 was used as an internal reference gene.

Figure 7

A

B

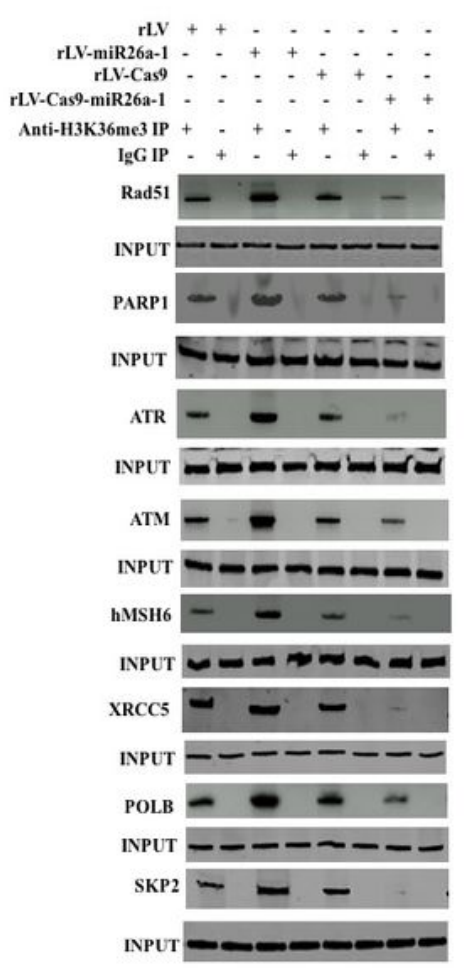

E

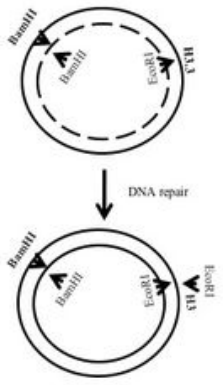

C

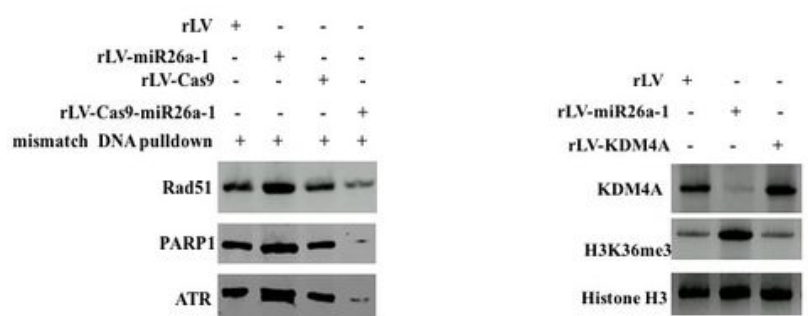

D

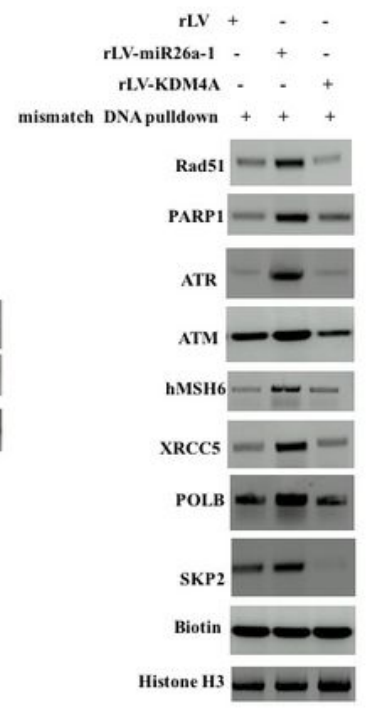

G

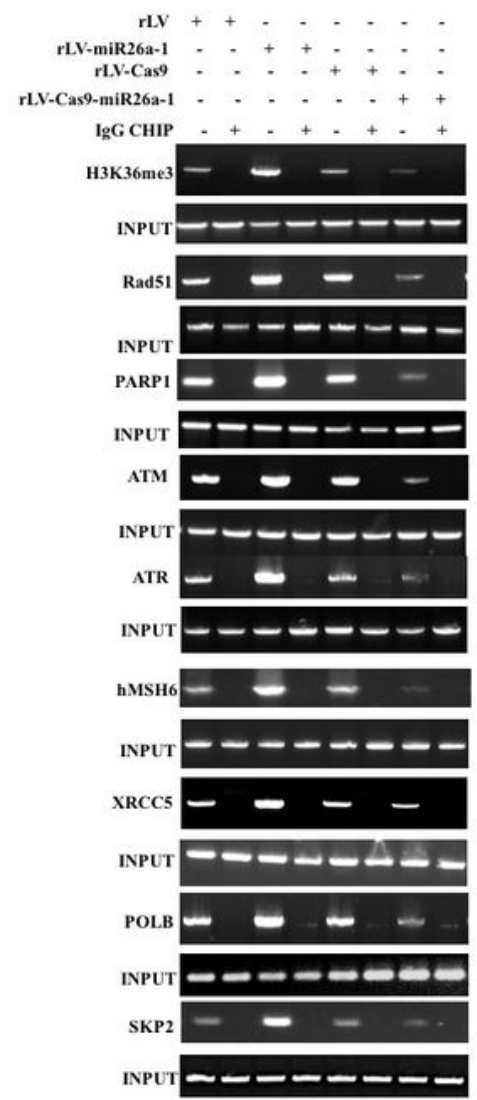

\section{Figure 7}

miR-26a-1 enhances the binding ability of Rad51, PARP1, ATR, ATM, hMSH6, XRCC5, POLB, SKP2 to mismatch DNA damage dependent on $\mathrm{H} 3 \mathrm{~K} 36 \mathrm{me}$, and promotes the formation of DNA damage repair complexes. A. Co- immunoprecipitation with anti-H3K36me3 and the precipitates were analyzed by 
Western blotting with anti-Rad51, anti-PARP1, anti-ATR, anti-ATM, anti-hMSH6, anti-XRCC5, anti-POLB, and anti-SKP2. B. DNA pulldown with Biotin- mismatched DNA damage probe and Western blotting analysis was performed with anti-Rad51, anti-PARP1, anti-ATR, anti-ATM, anti-hMSH6, anti-XRCC5, antiPOLB, and anti-SKP2. Western blotting with anti-Biotin as an internal reference (INPUT). C. Western blotting with anti-H3K36me3. HistoneH3 as an internal reference. D. DNA pulldown with Biotin mismatched DNA damage probes and Western blotting analysis was performed with anti-Rad51, antiPARP1, anti-ATR, anti-ATM, anti-hMSH6, anti-XRCC5, anti-POLB, and anti-SKP2. Western blotting with antiBiotin as an internal reference (INPUT). E. Plasmids with mismatched DNA.F. repeated chromosome immunoprecipitation (CHIP) with anti-Anti-Methylated Arg and anti-Anti-POLB. The DNA isolated and purified was used as a template, and the primers designed according to the plasmid sequence of the mismatched DNA are used for polymerase chain reaction (PCR) amplification. IgG CHIP is used as a negative control. G. The chromosomal immunoprecipitation (CHIP) with anti-Rad51, anti-PARP1, anti-ATR, anti-ATM, anti-hMSH6, anti-XRCC5, anti-POLB, anti-SKP2. The DNA isolated and purified from the CHIP precipitate is used as a template, and the primers designed according to the plasmid sequence of the mismatched DNA are used for polymerase chain reaction (PCR) amplification. IgG CHIP is used as a negative control. 
Figure 8

A

B

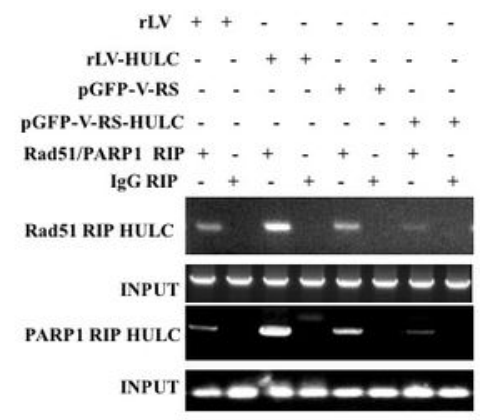

E

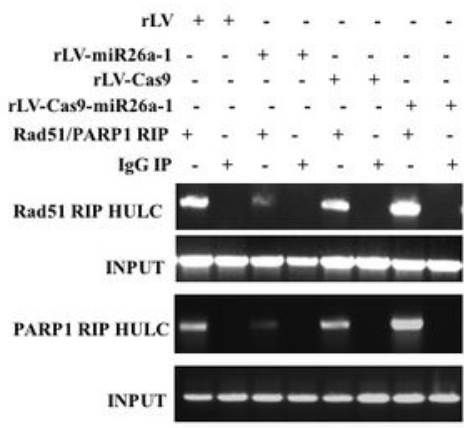

F

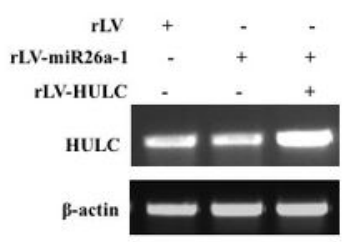

G

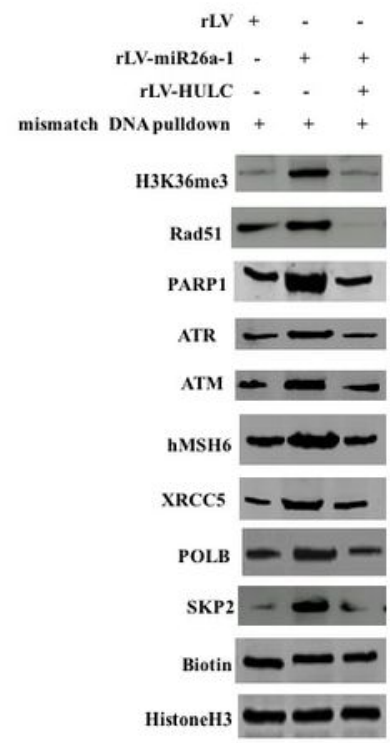

C

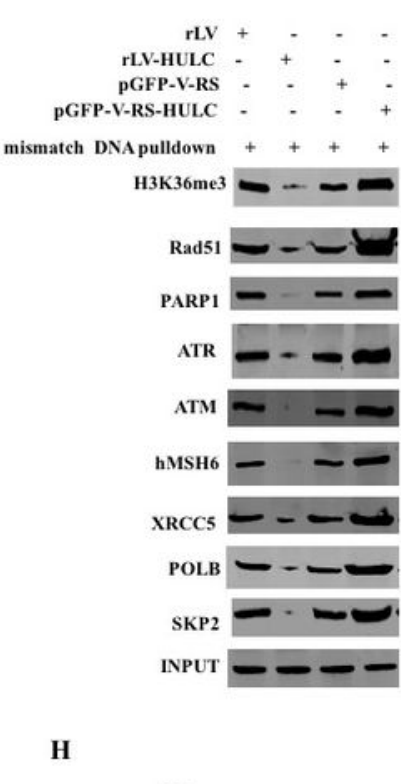

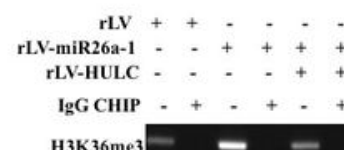

н3кзтез --
INPUT $-\square=-\square$

Rad51 - - -

INPUT $=-a-a$

PARP1 - - -

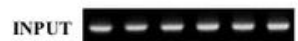

ATR - - -

INPUT

ATM - -

INPUT $-0-0-$

${ }_{\text {hMSH6 }}--$

INPUT $----\div$

XRcC5 --

INPUT - - - - -

POLB - $\quad-\quad$ -

INPUT $=--ー-\square$

SKP2 --

INPUTーーーーーー
D

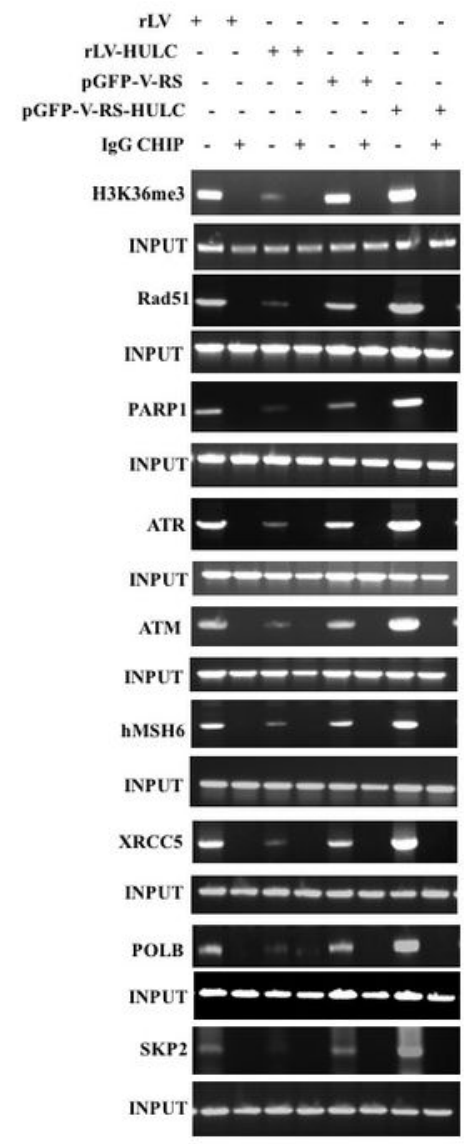

\section{Figure 8}

miR-26a-1 promotes the formation of DNA damage repair complexes dependent on long non-coding RNA HULC . A. Reverse polymerase chain reaction (RT-PCR) was used to detect the transcription ability of HULC. $\beta$-actin is used as an internal reference gene. B. RNA immunoprecipitation (RIP) with anti-PARP1 or anti-Rad51.The HULC was amplified by RT-PCR. IgG RNA immunoprecipitation was used as a negative control, and the RNA retained before RIP was used as a template to amplify HULC as an internal reference 
(INPUT).C. DNA pulldown with biotin- mismatched DNA damage probe and western blotting analysis was performed with anti-Rad51, anti-PARP1, anti-ATR, anti-ATM, anti-hMSH6, anti-XRCC5, anti-POLB, and antiSKP2 respectively. Anti-Biotin Western blotting is used as an internal reference (INPUT) for the amount of probe added. D. Chromosome immunoprecipitation (CHIP) with anti-H3K 36me3, anti-Rad51, anti-PARP1, anti-ATR, anti-ATM, anti-hMSH6, anti-XRCC5, anti-POLB, and anti-SKP2. The DNA isolated and purified from the CHIP precipitate is used as a template, and the polymerase chain reaction (PCR) amplification is carried out with primers designed according to the plasmid sequence of the mismatched DNA. E. RNA immunoprecipitation (RIP) with anti-PARP1 or anti-Rad. The HULC was amplified by RT-PCR with primers designed by the HULC sequence. IgG RNA immunoprecipitation was used as a negative control F. RT-PCR was used to detects the transcription ability of HULC. $\beta$-actin is used as an internal reference gene. G. DNA pull down with anti-Rad51, anti-PARP1, anti-ATR, anti-ATM, anti-hMSH6, anti-XRCC5, anti-POLB, and antiSKP2 respectively. Anti-Biotin Western blotting with anti-biotin was used as the internal reference (INPUT) . H. chromosome immunoprecipitation (CHIP) with anti-H3K36me3, anti-Rad51, anti-PARP1, anti-ATR, anti-ATM, anti-hMSH6, anti-XRCC5, anti-POLB, and anti-SKP2. The DNA isolated and purified from the CHIP precipitate is used as a template, and the polymerase chain reaction (PCR) amplification is carried out with primers designed according to the plasmid sequence of the mismatched DNA. IgG CHIP is used as a negative control; the DNA retained before chromatin immunoprecipitation is used as a template, and the product amplified by primers designed with a plasmid sequence of mismatched DNA is used as an internal reference (INPUT).

Figures
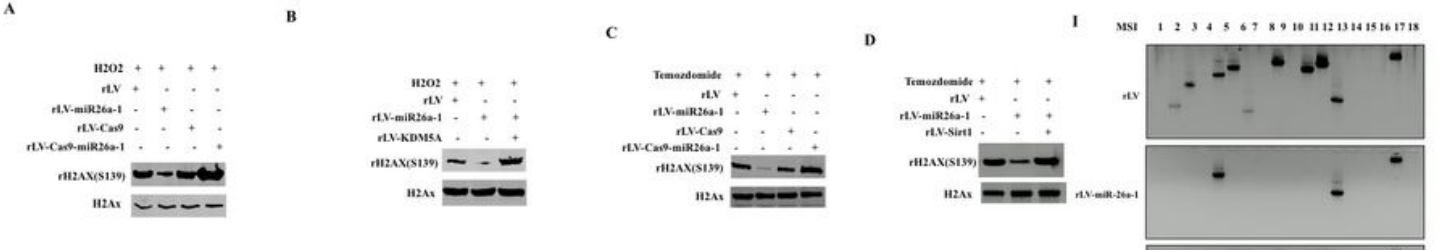

E
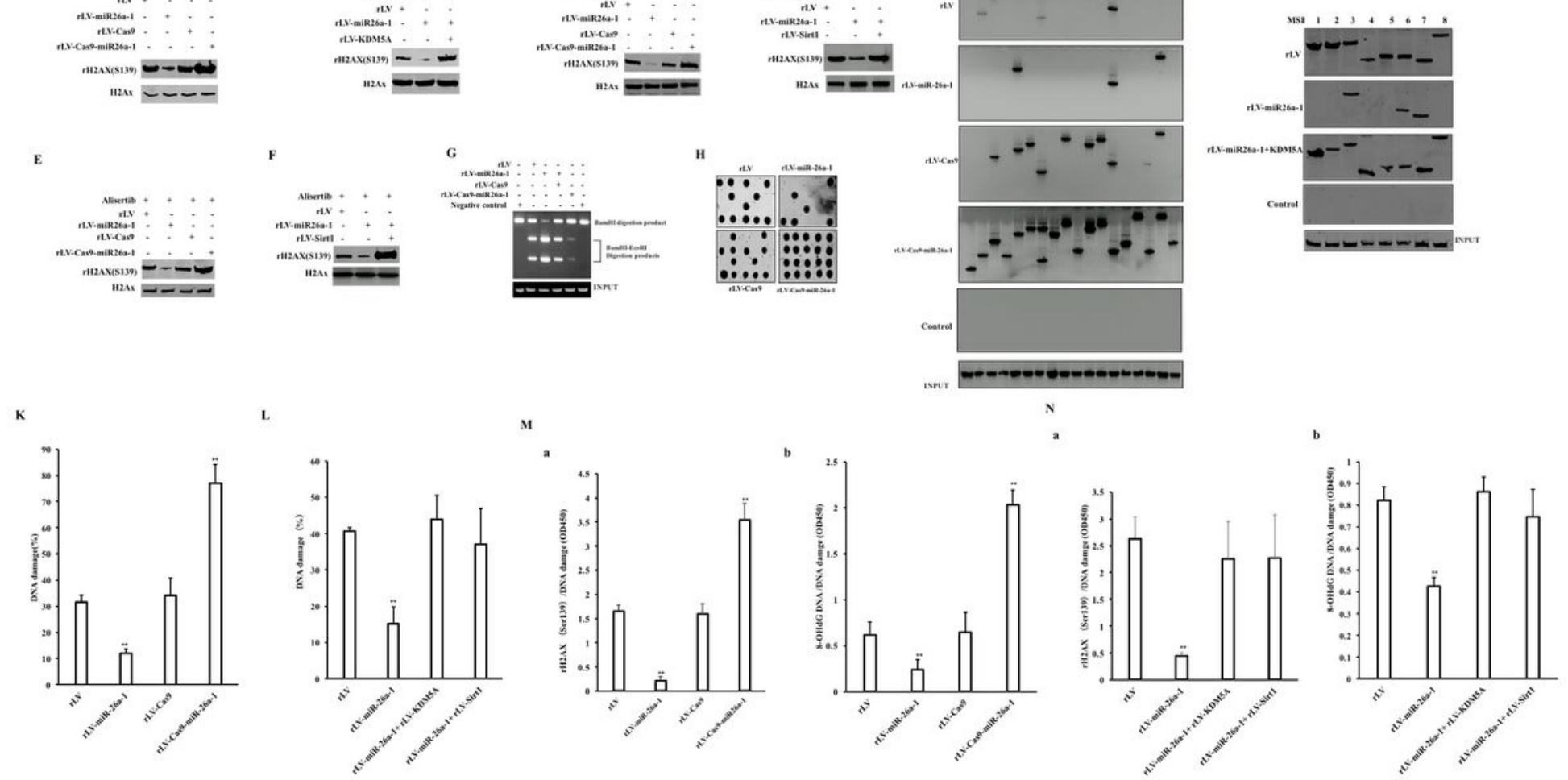

Figure 9 
miR-26a-1 promotes the DNA damage repair by inhibiting Sirt1 and KDM5A. A. Western blotting was used to detect the level of DNA damage marker rH2AX (S139). H2AX as an internal reference gene. B. Western blotting was used to detect the DNA damage marker rH2AX (S139). H2AX as an internal reference gene. C-D. Western blotting was used to detect the levels of DNA damage marker rH2AX (S139). H2AX as an internal reference gene. E. After treatment with Alisertib, Western blotting was used to detect the levels of DNA damage marker rH2AX (S139) .H2AX as an internal reference gene. F. Western blotting was used to detect the levels of DNA damage marker rH2AX (S139).H2AX as an internal reference gene. G. The plasmid was transfected with mismatched DNA, and then PCR combined with BamHI-EcoRI restriction analysis was used to detect the level of DNA damage repairs. H. After Alisertib induces cell DNA damage, and then Dot blot was used to detect the level of DNA damage repair I. After Alisertib was used to induce cellular DNA damage, and then Northern blot was used to detect the level of DNA microsatellite instability in these four stable cell lines. J. After Alisertib induces cell DNA damage, Northern blot was used to detect the level of DNA microsatellite instability. K. The immunostaining was used to detect the level of DNA damage marker $\mathrm{rH} 2 \mathrm{AX}$ (S139). Each experiment was repeated three times. Each group value is expressed as mean \pm standard deviation (mean $\pm S E M, n=3$ ), **, $P<0.01, *, P<0.05$. L. The immunostaining was used to detect the level of DNA damage marker rH2AX (S139). M-N. a. Cells were treated with DNA damage inducer Temozolomide and then in situ DNA damage analysis was performed. b. Quantitative analysis of DNA Damgae via 8-OHdG. Each experiment was repeated three times. Each group value is expressed as mean \pm standard deviation (mean \pm SEM, $n=3$ ), **, $P<0.01, *, P<0.05$.

Figure 10

A

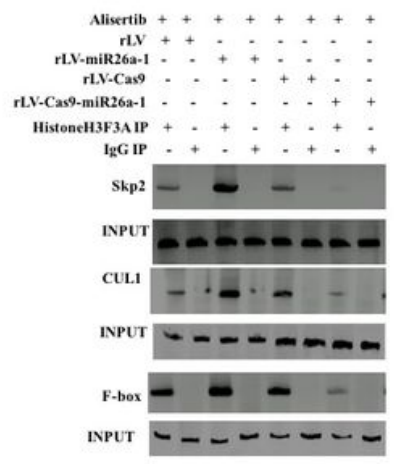

E

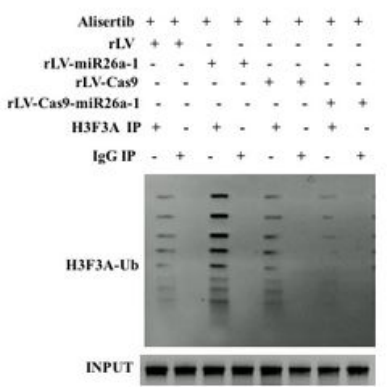

B
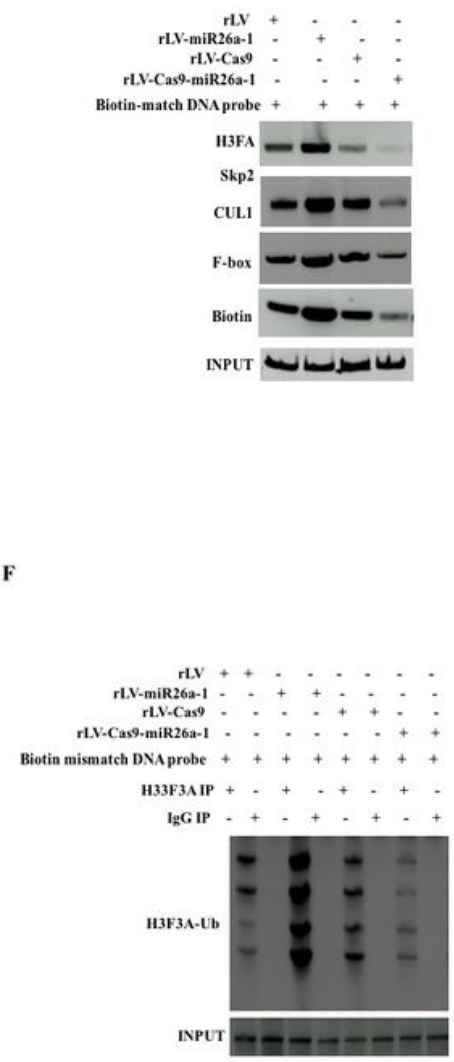

C

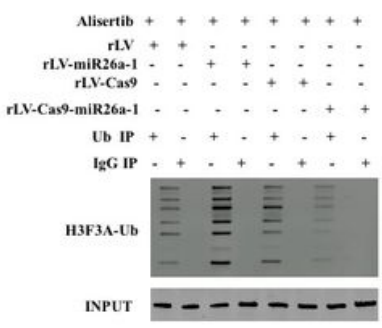

G

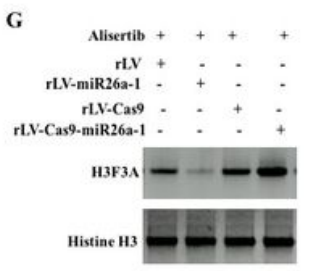

I

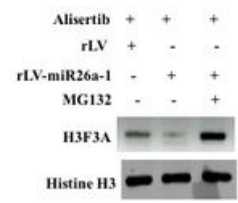

D
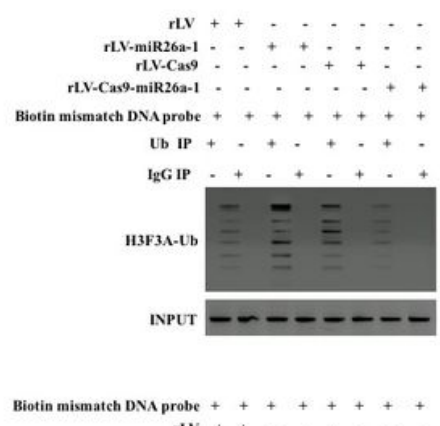

H

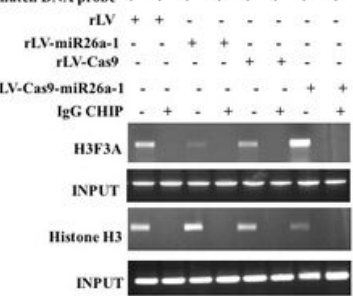

Biotin mismatch DNA probe ++++++

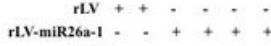

MG132 . . . + +

${ }_{\mathrm{g} G \mathrm{CHIP}}++\ldots+$

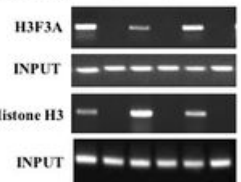




\section{Figure 10}

miR-26a-1 increases the renewal ability of histones dependent on protein ubiquitination degradation pathway after DNA damage repair. A. to Co- immunoprecipitation with Anti-H3FA and then Western blotting analysis with anti-Skp2, anti-CUL1, and anti-F-box. IgG immunoprecipitation was used as a negative control, and samples before coprecipitatio were detected by Western blotting with anti-H3F3A as INPUT. B. DNA pulldown experimente with Biotin labeled mismatched DNA damage probe. Western blotting analysis was performed with anti-H3F3A, anti-Skp2, anti-CUL1, and anti-F-box. Anti-Biotin Western blotting was used as the internal reference (INPUT) for the amount of probe added. C. Coimmunoprecipitation with Anti-Ub and then Western blotting analysis with anti-H3F3A. IgG immunoprecipitation was used as a negative control, and samples before coprecipitation were detected by Western blotting with anti-H3F3A as INPUT. D. DNA pulldown with Biotin labeled mismatched DNA damage probe. E. Co-immunoprecipitation with anti-H3F3A and then Western blotting analysis with anti-U b. F. DNA pulldown with biotin-labeled mismatch DNA damage probes. And then co-immunoprecipitation with anti-H3F3A, and analyze the precipitates with Western blotting with anti-Ub IgG immunoprecipitation was used as a negative control, and samples before coprecipitation were detected by Western blotting with anti-H3F3A as INPUT. G. Western blotting with anti-H3F3A. Anti-Histine H3 serves as the internal reference. H. Chromosomal immunoprecipitation (CHIP) with anti-H3F3A and anti-Histone H3. The DNA isolated and purified from the CHIP precipitate is used as a template, and the polymerase chain reaction (PCR) amplification is performed with primers designed according to the plasmid sequence of the repaired DNA. I. Western blotting with anti-H3F3A. Anti-Histine H3 serves as the internal reference. J. Chromosomal immunoprecipitation (CHIP) with anti-H3F3A and anti-Histone H3. The DNA isolated and purified from the CHIP precipitates are used as a template, and the polymerase chain reaction (PCR) amplification is performed with primers designed according to the plasmid sequence of the repaired DNA. 
Figure 11

A
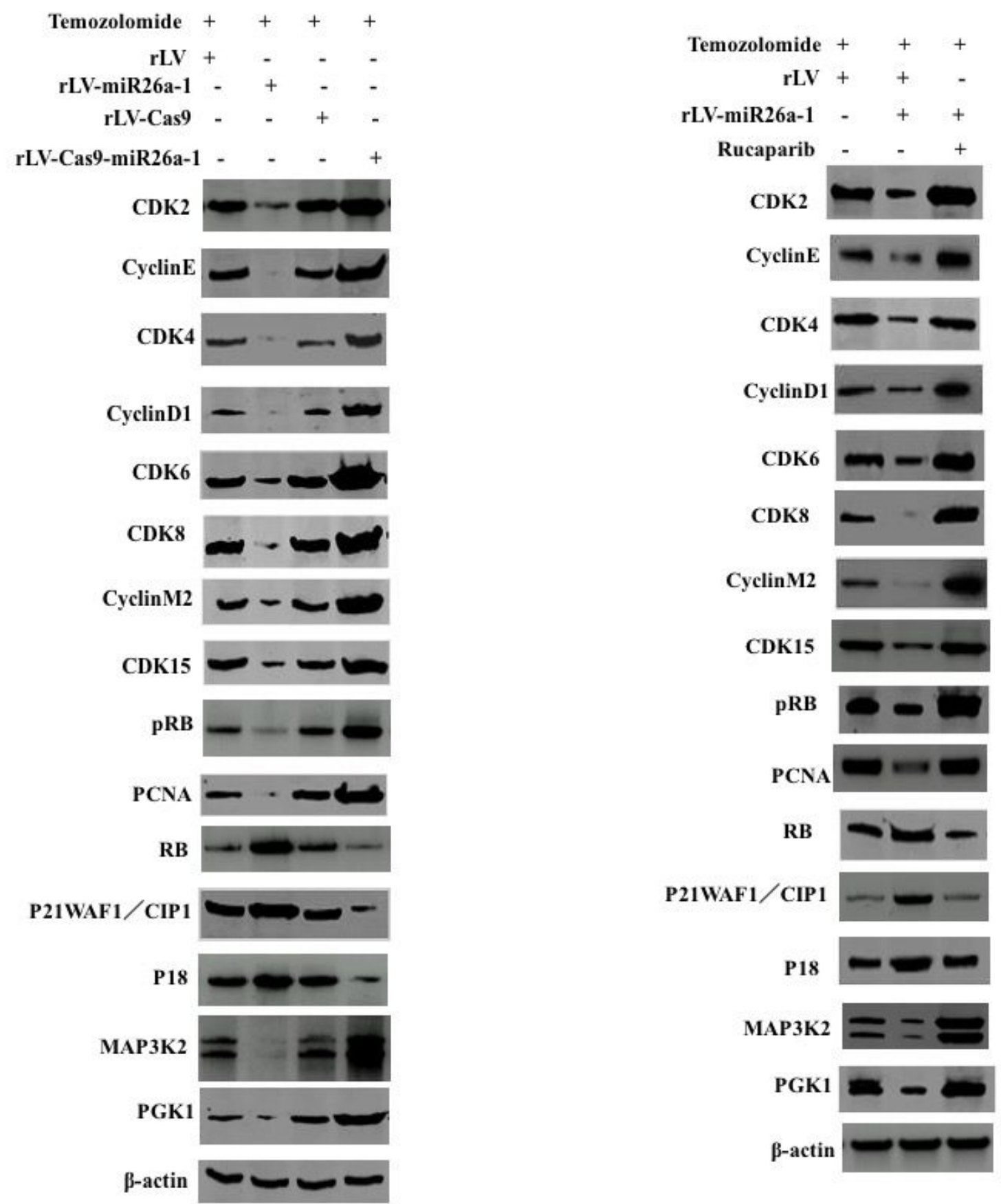

Figure 11

miR-26a-1 affects the expression of cyclin and protein kinase dependent on DNA damage repair in liver cancer stem cells. A. Western blotting was used to detect CDK2, CyclinE, CDK4, CyclinD1, CDK6, and CDK8 ,CyclinM2, CDK15, RB, pRB, PCNA, P18, P21/WAF1/Cip1, MAP3K2, and PGK1, $\beta$-actin as an internal reference gene. B. Western blotting was used to detect CDK2, CyclinE, CDK4, CyclinD1, CDK6, CDK8, 
CyclinM2, CDK15, RB, pRB, PCNA, P18, P21/WAF1/Cip1, MAP3K2, PGK1 . $\beta$-actin is used as an internal reference gene.

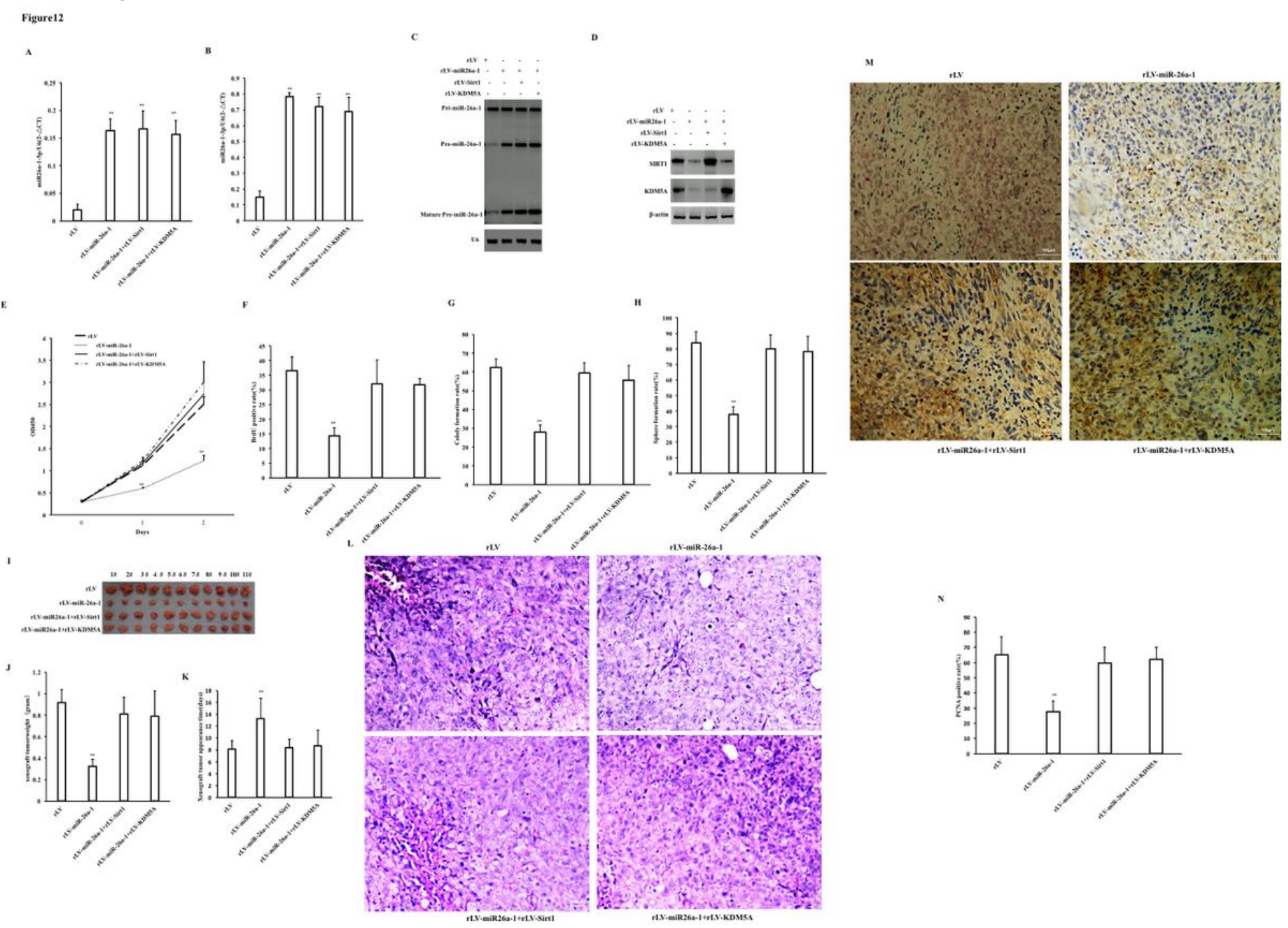

Figure 12

Excessive Sirt1 and KDM5A abolish miR-26a-1's ability to inhibit the growth of liver cancer stem cells. A. Quantitative reverse transcription polymerase chain reaction (RT-PCR) was used to detect the miR-26a-1$5 p$. U6 As an internal reference gene. B. Quantitative reverse transcription polymerase chain reaction (RTPCR) was used to detect the miR-26a-1-3p . U6 as the internal reference gene. C. Northern blotting was used to detect the precursors, precursors and mature miR-26a-1. U6 serves as an internal reference gene. D. The expression of Sirt1 or KDM5A were detected by Western blotting. $\beta$-actin serves as an internal reference. E. CCK8 cell growth assay analysis F. BrdU staining assay. G. Determination of plate colony forming ability. H. Determination of the cell spheres. I. Photograph of xenograft. J. Comparison of the size (grams) of xenograft tumors. K.Comparison of the time (days) of the appearance of transplanted tumors in nude mice. L. Transplant tumor tissue sections $(4 \mu \mathrm{m})$ of nude mice fixed in $4 \%$ formaldehyde and embedded in paraffin were stained with hematoxylin-eosin (HE) (original magnification×100). M. anti-PCNA immunohistochemical staining (original magnification×100). N. Comparison of PCNA positive rate. 


\section{Figure 13}

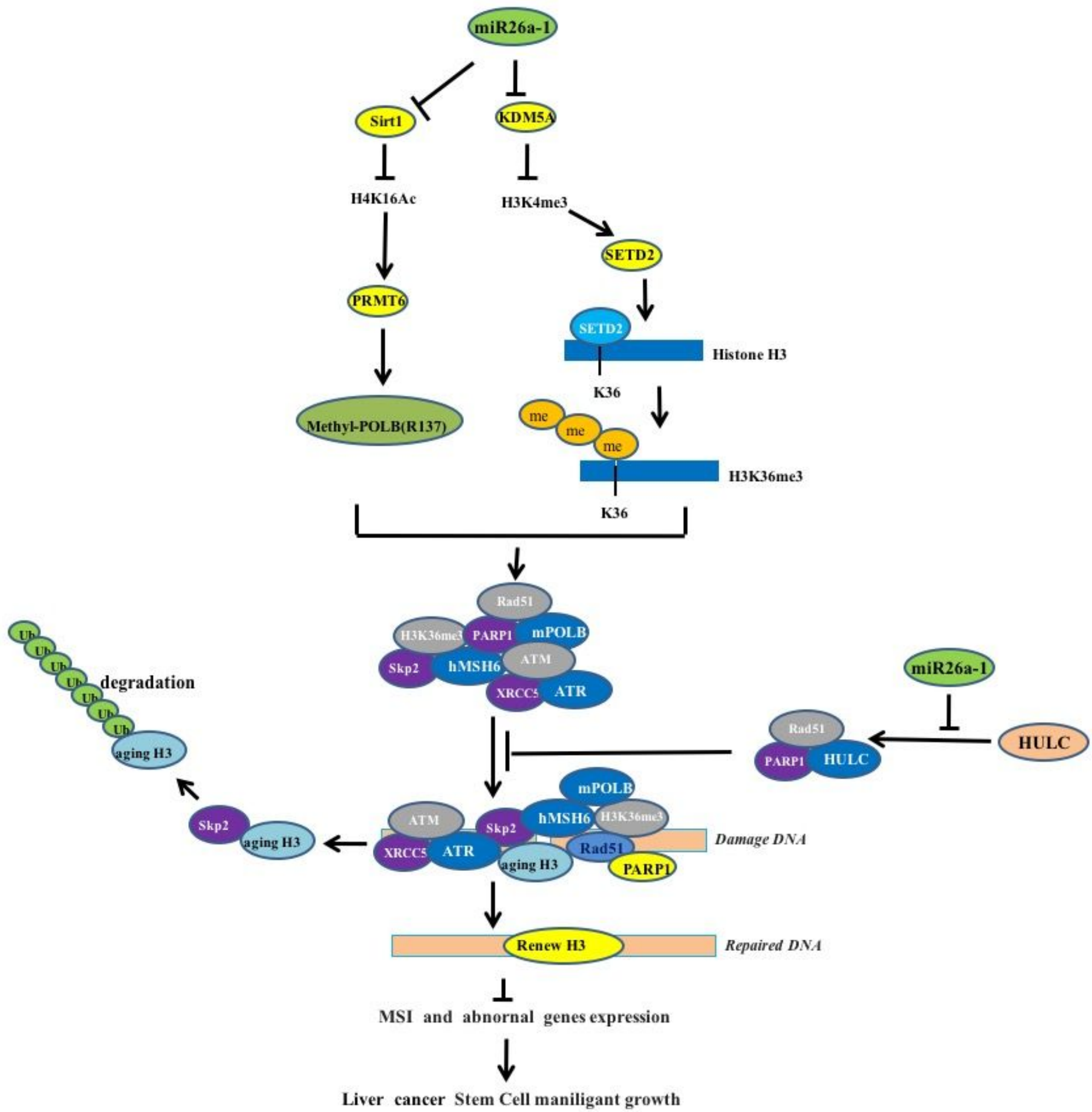

Figure 13

Schematic diagram of the molecular mechanism of miR-26a-1 inhibiting the growth of liver cancer stem cells.

\section{Supplementary Files}


This is a list of supplementary files associated with this preprint. Click to download.

- Supplemental.docx 